Mathematical Modelling And Analysis

Volume 21 Number 1, January 2016, 95-118

http://dx.doi.org/10.3846/13926292.2016.1137090

(C) Vilnius Gediminas Technical University, 2016
Publisher: Taylor\&Francis and VGTU

http://www.tandfonline.com/TMMA

ISSN: $1392-6292$

eISSN: 1648-3510

\title{
Proximal Point Algorithm for a Common of Countable Families of Inverse Strongly Accretive Operators and Nonexpansive Mappings with Convergence Analysis
}

\section{Khanittha Promluang ${ }^{a}$, Kanokwan Sitthithakerngkiet ${ }^{a, b}$ and Poom Kumam ${ }^{a, c, d}$}

${ }^{a}$ Department of Mathematics, Faculty of Science, King Mongkut's University of Technology Thonburi (KMUTT)

126 Pracha-Uthit Road, Bang Mod, 10140 Thung Khru, Bangkok, Thailand ${ }^{b}$ Nonlinear Dynamic Analysis Research Center, Department of Mathematics, Faculty of Applied Science, King Mongkut's University of North Bangkok (KMUTNB)

1518 Pracharat 1 Road, Wongsawang, 10800 Bangsue, Bangkok, Thailand ${ }^{c}$ Theoretical and Computational Science (TaCS) Center, Science Laboratory Building, Faculty of Science, King Mongkut's University of Technology Thonburi (KMUTT)

126 Pracha-Uthit Road, Bang Mod, 10140 Thung Khru, Bangkok, Thailand ${ }^{d}$ China Medical University

No. 91, Hsueh-Shih Road, 40402 Taichung, Taiwan

E-mail(corresp.): poom.kum@kmutt.ac.th

E-mail: k.promluang@gmail.com

E-mail: kanokwan.s@sci.kmutnb.ac.th

Received May 2, 2015; revised December 26, 2015; published online January 15, 2016

\begin{abstract}
In this paper, we investigate and analyze a proximal point algorithm via viscosity approximation method with error. This algorithm is introduced for finding a common zero point for a countable family of inverse strongly accretive operators and a countable family of nonexpansive mappings in Banach spaces. Our result can be extended to some well known results from a Hilbert space to a uniformly convex and 2-uniformly smooth Banach space. Finally, we establish the strong convergence theorems for the proximal point algorithm. Also, some illustrative numerical examples are presented.
\end{abstract}

Keywords: Proximal Point Algorithm; Zero point; inverse strongly accretive operator; nonexpansive mapping.

AMS Subject Classification: 47H10; 47H09. 


\section{Introduction}

The problems of finding a zero point for monotone operators play an important role in modern optimization and analysis. It can be related to many kinds of important problems, such as convex minimization problems, equilibrium problems, variational inequality problems and others. In order to approximate the solution to these problems, many authors have intensively studied the convergence of such problems in several setting spaces. Martinet [15] first introduced the proximal point algorithm $(P P A)$ in a Hilbert space $H$; for starting $x_{0} \in H$, a sequence $\left\{x_{n}\right\}$ is generated by

$$
x_{n+1}=J_{r_{n}}^{A}\left(x_{n}\right), \forall n \in \mathbb{N},
$$

where $J_{r_{n}}^{A}=\left(I+r_{n} A\right)^{-1}$ is the resolvent operator of $A$ with $I$ being the identity mapping and $\left\{r_{n}\right\} \subset(0, \infty)$ is a regularization sequence.

Later, Rockafellar [18] has studied the proximal point algorithm which generates a sequence $\left\{x_{n}\right\}$ according to the following algorithm:

$$
x_{n+1}=J_{r_{n}}^{A} x_{n}+e_{n}, \forall n \in \mathbb{N},
$$

where $\left\{e_{n}\right\}$ is a sequence of errors and $\left\{r_{n}\right\} \subset(0, \infty)$ is a sequence of regularization parameters. Rockafellar proved that if $A^{-1}(0) \neq \emptyset$, then the sequence $\left\{x_{n}\right\}$ converges weakly to a solution of a zero point of $A$.

In 2000, Moudafi [16] introduced the viscosity approximation method for finding fixed point of a nonexpansive mapping $S$ in a Hilbert space; for given $x_{0} \in C$, the sequence is defined by the following algorithm:

$$
x_{n+1}=\alpha_{n} f\left(x_{n}\right)+\left(1-\alpha_{n}\right) S x_{n}, \forall n \geq 0,
$$

where $f: C \rightarrow C$ is a contraction mapping and $\left\{\alpha_{n}\right\} \subseteq(0,1)$ satisfies some condition. This sequence converges strongly to a fixed point of $S$.

After that, $\mathrm{Xu}[20]$ developed the viscosity approximation method in both Hilbert and Banach spaces. Strong convergence theorems for zero points of monotone operators were established in a Banach space. Moreover, many authors constructed some approximation algorithms for some nonlinear variational inclusions in Hilbert spaces or Banach spaces by using the resolvent operator technique. In $[6,13,14]$, the $P P A$ was extended to the case of sum of two monotone operators.

In 2014, Eslamian [8] proposed Rockafellar's proximal point algorithm for finding a zero point of a finite family of monotone operators via viscosity method in a Hilbert space. Eslamian [8] proposed the following algorithm:

$$
x_{n+1}=a_{n, 0} f\left(x_{n}\right)+a_{n, 1} J_{r_{n}}^{T_{1}} x_{n}+a_{n, 2} J_{r_{n}}^{T_{2}} x_{n}+\ldots+a_{n, m} J_{r_{n}}^{T_{m}} x_{n}+e_{n}
$$

for all $n \geq 1$ where $\sum_{i=0}^{m} a_{n, i}=1$ and for each $i=1,2, \ldots, m, T_{i}$, are a finite family of monotone operators of $H$. If $\left\{a_{n, i}\right\},\left\{e_{n}\right\}$ and $\left\{r_{n}\right\}$ are positive sequences and satisfy some conditions, then the sequence $\left\{x_{n}\right\}$ converges strongly.

Nowadays, several iterative methods have been proposed and analyzed to find a common solution of two different fixed point problems, such as a fixed 
point problem of nonexpansive mapping, fixed point problem of variational (inclusions) inequalities and other. However, there are a few iterative methods for finding a common solution of two different infinite countable family of the fixed point problems.

In this paper, motivated by $[8,16]$, we are interested in the problem for finding a common solution of two different fixed point problems which are a common element of a zero point for an infinite countable family of $\beta$-inverse strongly accretive operators and a common fixed point of an infinite countable nonexpansive mappings in uniformly convex and 2-uniformly smooth Banach space. Our main result study these problems in uniformly convex and 2 -uniformly smooth Banach space such as the spaces $L_{p}, l_{p}$ or Sobolev space $W_{m}^{p}$ (where $p \geq 2$ ), which is more general than Hilbert space. In addition, our main theorem solves the problems for finding a common element of an infinite family of accretive operators, which is more general than the problem of finding a common element of a finite family of monotone operators. Consequently, the strong convergence theorem is obtained. Finally, we have studied the convergence analysis of the proximal point algorithm. Also, some illustrative numerical examples (using Matlab software) are presented.

\section{Preliminaries}

Let $E$ be a real Banach space and $E^{*}$ be the dual space of $E^{*}$. Let $\langle\cdot, \cdot\rangle$ be the pairing between $E$ and $E^{*}$. For all $x \in E$ and $x^{*} \in E^{*}$, let the value of $x^{*}$ at $x$ be denoted by $\left\langle x, x^{*}\right\rangle$. The normalized duality mapping $J: E \rightarrow 2^{E^{*}}$ is defined by

$$
J(x)=\left\{x^{*} \in E^{*}:\left\langle x, x^{*}\right\rangle=\|x\|^{2},\|x\|=\left\|x^{*}\right\|\right\}, \quad \forall x \in E .
$$

A single-value normalized duality mapping is denoted by $j$, which means that a mapping $j: E \rightarrow E^{*}$ such that, for all $u \in E, j(u) \in E^{*}$ satisfies the following:

$$
\langle j(u), u\rangle=\|j(u)\|\|u\|, \quad\|j(u)\|=\|u\| .
$$

If $E=H$ is a Hilbert space, then $J=I$, where $I$ is identity mapping. If $E$ is a smooth Banach space, then $J$ is single-valued.

A Banach space $E$ is said to satisfy Opial's condition if for each sequence $\left\{x_{n}\right\}_{n=0}^{\infty}$ in $E$ such that $\left\{x_{n}\right\}$ converges weakly to some $x$ in $E$, the inequality

$$
\limsup _{n \rightarrow \infty}\left\|x_{n}-x\right\|<\limsup _{n \rightarrow \infty}\left\|x_{n}-y\right\|
$$

holds for all $y \in E$ with $y \neq x$. In fact, a Banach space with a weakly sequentially continuous duality mapping has the Opial's condition; see [9]. We known that if $E$ admits a weakly sequentially continuous duality mapping, then $E$ is smooth. It is well known that all Hilbert spaces and $l_{p},(p>1)$ satisfy Opial's condition, while $L_{p}$ does not satisfy this condition except $p=2$.

A Banach space $E$ is called a strictly convex if it satisfies the following condition

$$
\|x\|=\|y\|=\|(1-\lambda) x+\lambda y\|, \forall x, y \in E \text { and } 0<\lambda<1 \quad \Longrightarrow \quad x=y .
$$


Let $S(E)=\{x \in E:\|x\|=1\}$ be the unit sphere of $E$. $E$ is said to be uniformly convex if for each $\epsilon \in(0,2]$ there exists a constant $\delta=\delta(\epsilon)>0$ such that for all $x, y \in S(E)$, if $\|x-y\| \geq \epsilon$ then $\left\|\frac{x+y}{2}\right\| \leq 1-\delta$. It is well known that uniformly convex is strictly convex.

A Banach space $E$ is said to be smooth if the limit

$$
\lim _{t \rightarrow 0} \frac{\|x+t y\|-\|x\|}{t}
$$

exists for all $x, y \in S(E)$. In this case, the norm $\|\cdot\|$ of $E$ is said to be Gâteaux differentiable norm.

The norm of $E$ is said to be uniformly Gâteaux differentiable norm if for each $y \in S(E)$, the limit (2.1) is attained uniformly for all $x \in S(E)$ and it is said to be Freéchet differentiable if for each $x \in S(E)$, the limit (2.1) is attained uniformly for all $y \in S(E)$. Moreover, it is said to be uniformly smooth if the limit $(2.1)$ is attained uniformly for all $(x, y) \in S(E) \times S(E)$. It is well known that if the norm of $E$ is smooth, then the duality mapping $J$ is single-valued and norm to weak* continuous on a bounded subset of $E$.

The modulus of smoothness of $E$ is the function $\rho:[0, \infty) \rightarrow[0, \infty)$ defined by $\rho(t)=\sup \left\{\frac{1}{2}(\|x+y\|+\|x-y\|)-1: x, y \in E,\|x\|=1,\|y\|=t\right\}$. A Banach space $E$ is an uniformly smooth if and only if $\lim _{t \rightarrow 0} \frac{\rho(t)}{t}=0$.

A Banach space $E$ is said to be q-uniformly smooth if for $1<q \leq 2$ be a fixed real number, there exists a constant $c>0$ such that $\rho(t) \leq c t^{q}$ for all $t>0$. In the case $q=2, E$ is said to be 2-uniformly smooth if there exists a constant $c>0$ such that $\rho(t) \leq c t^{2}$ for all $t>0$. Typical examples of both uniformly convex and 2-uniformly smooth Banach space are $l_{p}, L_{p},(p \geq 2)$, the Sobolev space $W_{m}^{p},(p \geq 2)$ and all Hilbert space.

Recall that every uniformly smooth space is smooth [5]. If $E$ is a q-uniformly smooth, then $E$ is uniformly smooth. Hence 2 - uniformly smooth of Banach space is a uniformly smooth and it is uniformly Gâteaux differentiable norms. If $E$ is uniformly Gâteaux differentiable norms, then the duality mapping $J$ : $E \rightarrow 2^{E^{*}}$ is a single-valued and $J$ is norm to weak* uniformly continuous on a bounded subset of $E$.

Let $C$ be a nonempty closed convex subset of a Banach space $E$ and $D \subset C$, then a mapping $Q: C \rightarrow D$ is said to be sunny if $Q(x+t(x-Q(x)))=Q(x)$ whenever $Q x+t(x-Q(x)) \in C$ for all $x \in C$ and $t \geq 0$.

A mapping $Q: C \rightarrow C$ is called a retraction if $Q^{2}=Q$. Note that if a mapping $Q$ is a retraction, then $Q z=z$ for all $z \in R(Q)$ where $R(Q)$ is the range of $Q$. A subset $D$ of $Q$ is called a sunny nonexpansive retract of $C$ if there exists a sunny nonexpansive retraction from $C$ onto $D$.

Lemma 1. [17] Let $E$ be a smooth Banach space and let $C$ be a nonempty subset of $E$. Let $Q: E \rightarrow C$ be a retraction and let $J$ be the normalized duality mapping on $E$. Then, the following statements are equivalent:

(i) $Q$ is sunny and nonexpansive;

(ii) $\|Q x-Q y\|^{2} \leq\langle x-y, J(Q x-Q y)\rangle, \forall x, y \in E$;

(iii) $\|(x-y)-(Q x-Q y)\|^{2} \leq\|x-y\|^{2}-\|Q x-Q y\|^{2}$; (iv) $\langle x-Q x, J(y-$ $Q x)\rangle \leq 0, \forall x \in E, y \in C$. 
Lemma 2. [11] Let $C$ be a nonempty closed convex subset of a uniformly convex and uniformly smooth Banach space $E$ and let $S$ be a nonexpansive mapping of $C$ into itself with $F i x(S) \neq \emptyset$. Then, the set Fix $(S)$ is a sunny nonexpansive retract of $C$.

In 2004, $\mathrm{Xu}$ [20] studied the following continuous scheme:

$$
x_{t}=t f\left(x_{t}\right)+(1-t) S x_{t},
$$

where $f$ is a $k$-contraction and $S$ is nonexpansive mapping. On a uniformly smooth Banach space, $\mathrm{Xu}[20]$ proved that the sequence $\left\{x_{t}\right\}$ converges strongly to a fixed point of $S$. If we defines mapping $Q: \Pi_{c} \rightarrow F i x(S)$, where $\Pi_{c}$ denote the set of $k$-contraction, by $Q(f):=\lim _{t \rightarrow \infty} x_{t}$, then $Q(f)$ solves the following variational inequality:

$$
\langle(I-f) Q(f), j(Q(f)-p)\rangle \leq 0, \forall p \in F i x(S) .
$$

It well known that if $E=H$ is a Hilbert space, then a sunny nonexpansive retraction $Q_{C}$ is coincident with the metric projection from $E$ onto $C$, that is $Q_{C}=P_{C}$. Let $C$ be a nonempty closed convex subset of a smooth Banach space $E$ and let $x \in E$ and let $x_{0} \in C$. Then we have from Lemma 1 that $x_{0}=Q_{C} x$ if and only if $\left\langle x-x_{0}, J\left(y-x_{0}\right)\right\rangle \leq 0$ for all $y \in C$, where $Q_{C}$ is a sunny nonexpansive retraction from $E$ onto $C$.

Let $C$ be a nonempty closed convex subset of $E$. Recall the following definitions:

1. Let $f: C \rightarrow E$ be an operator. Then, $T$ is called $k$-contraction if there exists a coefficient $k(0<k<1)$ such that

$$
\|f x-f y\| \leq k\|x-y\|, \quad \forall x, y \in C \text {. }
$$

2. Let $S: C \rightarrow E$ be an operator. Then, $S$ is called nonexpansive if

$$
\|S x-S y\| \leq\|x-y\|, \quad \forall x, y \in C .
$$

3. Let $A: C \rightarrow E$ be an operator. Then, $A$ is called accretive if there exists $j(x-y) \in J(x-y)$ such that

$$
\langle A x-A y, j(x-y)\rangle \geqslant 0, \quad \forall x, y \in C .
$$

4. Let $A: C \rightarrow E$ be an operator. Then, $A$ is called $\beta$-inverse-strongly accretive if there exists a constant $\beta>0$ and $j(x-y) \in J(x-y)$ such that

$$
\langle A x-A y, j(x-y)\rangle \geq \beta\|A x-A y\|^{2}, \quad \forall x, y \in C .
$$

An accretive operator $A$ is said to be maximal accretive if there is no proper accretive extension of $A$. If $A$ is an m-accretive operator then $A$ is said to be maximal accretive [7]. In a real Hilbert space, an accretive operator is called monotone. Moreover, $A$ is called maximal monotone if $R(I+r A)=H$. In this paper, we use $A^{-1}(0)$ to denote the set of zeros of $A$. For an accretive operator $A$, we can define a nonexpansive single-valued mapping $J_{r}^{A}: R(I+r A) \rightarrow D(A)$ by $J_{r}^{A}=(I+r A)^{-1}$ for each $r>0$, which is called the resolvent of $A$. It is known that $0 \in A(x) \Leftrightarrow x \in F i x\left(J_{r}^{A}\right)$.

In the sequel to give our main results, we need the following lemmas. 
Lemma 3. [4] Let $C$ be a closed convex subset of a strictly convex Banach space $E$. Let $T_{i}: C \rightarrow C$ be nonexpansive mappings for each $i \geq 1$ such that $\bigcap_{i=1}^{\infty} \operatorname{Fix}\left(T_{i}\right)$ is nonempty. Let $\left\{\alpha_{i}\right\}$ be a real number sequence in $(0,1)$ such that $\sum_{i=1}^{\infty} \alpha_{i}=1$. Then, the mapping $\dot{S}$ on $C$ defined by $S^{\prime} x=\sum_{i=1}^{\infty} \alpha_{i} T_{i} x$ for all $x \in C$ is well defined. Moreover, the mapping $\dot{S}$ is nonexpansive mapping and $\operatorname{Fix}(\dot{S})=\bigcap_{i=1}^{\infty} \operatorname{Fix}\left(T_{i}\right)$.

Remark 1. For each $n \geq 1$, if we set $T_{i}=S_{n} J_{r_{n}}^{A_{i}}$ then $T_{i}$ is nonexpansive mappings for all $i \geq 1$. Indeed, since $S_{n}$ and $J_{r_{n}}^{A_{i}}$ are nonexpansive mappings, then we obtain that

$$
\begin{aligned}
\left\|T_{i} x-T_{i} y\right\| & =\left\|S_{n} J_{r_{n}}^{A_{i}} x-S_{n} J_{r_{n}}^{A_{i}} y\right\|, \forall n \in \mathbb{N} \\
& \leq\left\|J_{r_{n}}^{A_{i}} x-J_{r_{n}}^{A_{i}} y\right\|, \forall i \in \mathbb{N} \leq\|x-y\| .
\end{aligned}
$$

Thus, $T_{i}=S_{n} J_{r_{n}}^{A_{i}}$ is a nonexpansive mapping for each $n \geq 1$.

Lemma 4. [19] Let $q \in(1, \infty), \lambda \in[0,1]$ and $W_{q}(\lambda):=\lambda^{q}(1-\lambda)+\lambda(1-\lambda)^{q}$. Let $E$ be a real q-uniformly smooth Banach space. Then, there exist constants $c_{q}>0$ such that for all $x, y \in E$, the following inequality holds:

$$
\|\lambda x+(1-\lambda) y\|^{q} \leq \lambda\|x\|^{q}+(1-\lambda)\|y\|^{q}-W_{q}(\lambda) c_{q}\|x-y\|^{q} .
$$

Lemma 5. Let $E$ be a 2-uniformly smooth of a real Banach space. Then, for each $x_{i} \in E, \alpha_{i} \in[0,1], i \in \mathbb{N}$ with $\sum_{i=1}^{\infty} \alpha_{i}=1$, we have

$$
\left\|\sum_{i=1}^{\infty} \alpha_{i} x_{i}\right\|^{2} \leq \sum_{i=1}^{\infty} \alpha_{i}\left\|x_{i}\right\|^{2} .
$$

Proof. We will prove this lemma by the mathematical induction.

(1) If $k=2$, by using Lemma 4, we have

$$
\left\|\alpha_{1} x_{1}+\alpha_{2} x_{2}\right\|^{2} \leq \alpha_{1}\left\|x_{1}\right\|^{2}+\left(1-\alpha_{1}\right)\left\|x_{2}\right\|^{2} .
$$

Hence, the conclusion holds.

(2) Suppose that the inequality (2.2) holds for $k=n-1$, i.e.,

$$
\left\|\sum_{i=1}^{n-1} \alpha_{i} x_{i}\right\|^{2} \leq \sum_{i=1}^{n-1} \alpha_{i}\left\|x_{i}\right\|^{2} .
$$

We want to show that the inequality holds for $k=n$. Let $\alpha_{n} \neq 1$ be chosen in such a way that $\sum_{i=1}^{\infty} \alpha_{i}=1$. It follows from the induction hypotheses that

$$
\begin{aligned}
\left\|\sum_{i=1}^{n} \alpha_{i} x_{i}\right\|^{2} & =\left\|\sum_{i=1}^{n-1} \alpha_{i} x_{i}+\alpha_{n} x_{n}\right\|^{2} \\
& =\left\|\left(1-\alpha_{n}\right) \frac{\sum_{i=1}^{n-1} \alpha_{i} x_{i}}{\left(1-\alpha_{n}\right)}+\alpha_{n} x_{n}\right\|^{2} \\
& \leq\left(1-\alpha_{n}\right)\left\|\frac{\sum_{i=1}^{n-1} \alpha_{i} x_{i}}{\left(1-\alpha_{n}\right)}\right\|^{2}+\alpha_{n}\left\|x_{n}\right\|^{2} \\
& \leq \sum_{i=1}^{n-1} \alpha_{i}\left\|x_{i}\right\|^{2}+\alpha_{n}\left\|x_{n}\right\|^{2} \leq \sum_{i=1}^{n} \alpha_{i}\left\|x_{i}\right\|^{2} .
\end{aligned}
$$


This completes the proof.

Lemma 6. [19] Let E be a 2-uniformly smooth Banach space. Then, for any $x, y, \in E$, the following inequality holds

$$
\|x+y\|^{2} \leq\|x\|^{2}+2\langle y, j(x)\rangle+2\|K y\|^{2}, \quad j(x) \in J(x),
$$

where $K$ is the 2-uniformly smooth constant of $E$.

Lemma 7. [10] Let $E$ be an real Banach space and $J$ the normalized duality map on $E$. Then, for any $x, y, \in E$, the following inequality holds;

$$
\|x+y\|^{2} \leq\|x\|^{2}+2\langle y, j(x+y)\rangle, \forall j(x+y) \in J(x+y) .
$$

Lemma 8. Let $C$ be a nonempty closed convex subset of a 2-uniformly smooth Banach space $E$ with the 2-uniformly smooth constant $K$. Let $A$ be an $\beta$-inverse strongly accretive operator of $C$ into $E$ with a constant $\beta>0$. Then, we have

$$
\left\|J_{r}^{A} x-J_{r}^{A} y\right\|^{2} \leq\|x-y\|^{2}-2\left(\frac{\beta}{r}-K^{2}\right)\left\|\left(x-J_{r}^{A} x\right)-\left(y-J_{r}^{A} y\right)\right\|^{2} .
$$

In particular, if $r \in\left(0, \frac{\beta}{K^{2}}\right)$, then $J_{r}^{A}$ is a nonexpansive.

Proof. Let $x, y \in C$. By using Lemma 6, we obtain that

$$
\begin{aligned}
\left\|J_{r}^{A} x-J_{r}^{A} y\right\|^{2} & =\left\|(x-y)-(x-y)+J_{r}^{A} x-J_{r}^{A} y\right\|^{2} \\
& =\left\|(x-y)-\left(\left(x-J_{r}^{A} x\right)-\left(y-J_{r}^{A} y\right)\right)\right\|^{2} \\
& =\|(x-y)-r(A x-A y)\|^{2} \\
& \leq\|x-y\|^{2}-2 r\langle A x-A y, j(x-y)\rangle+2 K^{2} r^{2}\|A x-A y\|^{2} \\
& \leq\|x-y\|^{2}-2 r \beta\|A x-A y\|^{2}+2 K^{2} r^{2}\|A x-A y\|^{2} \\
& \leq\|x-y\|^{2}-2 r\left(\beta-K^{2} r\right)\|A x-A y\|^{2} .
\end{aligned}
$$

Moreover, if $r \in\left(0, \frac{\beta}{K^{2}}\right)$, we obtain that

$$
\begin{aligned}
\left\|J_{r}^{A} x-J_{r}^{A} y\right\|^{2} & \leq\|x-y\|^{2}-2 r\left(\alpha-K^{2} r\right)\left\|\frac{1}{r}\left(x-J_{r}^{A} x\right)-\frac{1}{r}\left(y-J_{r}^{A} y\right)\right\|^{2} \\
& \leq\|x-y\|^{2}-2 r\left(\beta-K^{2} r\right) \frac{1}{r^{2}}\left\|\left(x-J_{r}^{A} x\right)-\left(y-J_{r}^{A} y\right)\right\|^{2} \\
& \leq\|x-y\|^{2}-2\left(\frac{\beta}{r}-K^{2}\right)\left\|\left(x-J_{r}^{A} x\right)-\left(y-J_{r}^{A} y\right)\right\|^{2} \\
& \leq\|x-y\|^{2} .
\end{aligned}
$$

Thus, if $r \in\left(0, \frac{\beta}{K^{2}}\right)$, then $J_{r}^{A}$ is a nonexpansive mapping.

Lemma 9. [2] (The Resolvent Identity) For all $r>0, s>0$ and $x \in E$, then

$$
J_{r}^{B} x=J_{s}^{B}\left(\frac{s}{r} x+\left(1-\frac{s}{r}\right) J_{r}^{B} x\right) .
$$


Lemma 10. [3] (Demiclosed principle) Let $C$ be a nonempty closed convex subset of a uniformly convex Banach space $E$ and $S: C \rightarrow C$ be a nonexpansive mapping. Then $I-S$ is demiclosed at zero, i.e., $x_{n} \rightarrow x$ and $x_{n}-S x_{n} \rightarrow 0$ imply $x=S x$.

Lemma 11. [1] Let $C$ be a nonempty closed convex subset of a Banach space $E$ and let $\left\{S_{0}, S_{1}, S_{2}, \ldots\right\}$ be a sequence of mappings of $C$ into itself. Suppose that $\sum_{n=1}^{\infty} \sup \left\{\left\|S_{n+1} x-S_{n} x\right\|: x \in C\right\}<\infty$. Then, for each $y \in C,\left\{S_{n} y\right\}$ strongly converges to some point of $C$. Moreover, if $S$ is a mapping of $C$ into itself defined by $S y=\lim _{n \rightarrow \infty} S_{n} y, \forall y \in C$. Then, $\lim _{\sup _{n \rightarrow \infty}}\left\{\left\|S x-S_{n} x\right\|\right.$ : $x \in C\}=0$.

Lemma 12. [12] Assume that $\left\{a_{n}\right\}$ is a sequence of nonnegative real numbers satisfying the condition

$$
a_{n+1} \leq\left(1-t_{n}\right) a_{n}+t_{n} b_{n}+c_{n}, \forall n \geq 0,
$$

where $\left\{t_{n}\right\}$ is a sequence in $(0,1)$ such that $\lim _{n \rightarrow \infty} t_{n}=0$ and $\sum_{n \rightarrow \infty}^{\infty} t_{n}=\infty$, $\left\{b_{n}\right\}$ is a sequence such that $\lim _{\sup _{n \rightarrow \infty}} b_{n} \leq 0$ and $\left\{c_{n}\right\}$ is a positive number sequence such that $\sum_{n \rightarrow \infty}^{\infty} c_{n}<\infty$. Then, $\lim _{n \rightarrow \infty} a_{n}=0$.

\section{Main Result}

Theorem 1. Let $E$ be a uniformly convex and 2-uniformly smooth of real Banach space and let $C$ be a nonempty closed convex subset of $E$ which has a weakly continuous duality mapping $J$ from $E$ to $E^{*}$. For any $i \in \mathbb{N}$, let $\left\{A_{i}\right\}_{i=1}^{\infty}$ be an infinite sequence of $\beta_{i}$-inverse strongly accretive operators in $E$ such that $\beta:=\inf _{i \geq 1}\left\{\beta_{i}\right\}>0$ and $\bigcap_{i=1}^{\infty} \overline{D\left(A_{i}\right)} \subseteq C \subseteq \bigcap_{i=1}^{\infty} R\left(I+r A_{i}\right)$ for all $r>0$. Let $\left\{S_{n}\right\}_{n=1}^{\infty}$ be an infinite sequence of nonexpansive mapping from $C$ into itself such that $F:=\left(\bigcap_{n=1}^{\infty} \operatorname{Fix}\left(S_{n}\right)\right) \bigcap\left(\bigcap_{i=1}^{\infty} A_{i}^{-1}(0)\right) \neq \emptyset^{1}$. Let $f: C \rightarrow C$ be a contraction mapping with the constant $k \in(0,1)$ and $J_{r_{n}}^{A_{i}}=\left(I+r_{n} A_{i}\right)^{-1}$ be a resolvent of $A_{i}$ for each $r_{n}>0$. For given $x_{1} \in \bigcap_{i=1}^{\infty} \overline{D\left(A_{i}\right)}$, let $\left\{x_{n}\right\}$ be a sequence defined by the following:

$$
x_{n+1}=\alpha_{n, 0} f\left(x_{n}\right)+\sum_{i=1}^{\infty} \alpha_{n, i} S_{n} J_{r_{n}}^{A_{i}} x_{n}+e_{n}, \quad \forall n \geq 1,
$$

where $\sum_{i=0}^{\infty} \alpha_{n, i}=1,\left\{\alpha_{n, i}\right\} \subset(0,1)$ for all $i \geq 0,\left\{r_{n}\right\} \subset\left(0, \frac{\beta}{K^{2}}\right)$ where $K$ is the 2-uniformly smooth constant and $\left\{e_{n}\right\} \subset(0, \infty)$. Suppose that these sequences satisfy the following conditions:

(a) $\lim _{n \rightarrow \infty} \alpha_{n, 0}=0, \sum_{n=1}^{\infty} \alpha_{n, 0}=\infty$ and $\sum_{n=1}^{\infty}\left|\alpha_{n+1,0}-\alpha_{n, 0}\right|<\infty$;

(b) for each $i \geq 1, \lim _{n \rightarrow \infty} \alpha_{n, i}=\alpha_{i} \in(0,1)$;

(c) $\sum_{n=1}^{\infty}\left|r_{n+1}-r_{n}\right|<\infty$ and $\lim _{n \rightarrow \infty} r_{n}=r>0$;

${ }^{1}$ For example, we set $S_{n} x=\frac{x}{n+1}$ for all $n \geq 1$ and $A_{i} x=i x$ for all $i \geq 1$. Note the $F \neq \emptyset$ because $0 \in \bigcap_{n=1}^{\infty} \operatorname{Fix}\left(S_{n}\right)$ and $0 \in \bigcap_{i=1}^{\infty} A_{i}^{-1}(0)$. 
(d) $\sum_{n=1}^{\infty} e_{n}<\infty$

Assume that $\sum_{n=1}^{\infty} \sup \left\{\left\|S_{n+1} x-S_{n} x\right\|: x \in B\right\}<\infty$ for any bounded subset $B$ of $C$. Let $S$ be a mapping of $C$ into itself defined by $S x=\lim _{n \rightarrow \infty} S_{n} x$ for all $x \in C$ such that Fix $(S)=\bigcap_{n=1}^{\infty} F i x\left(S_{n}\right)$. Then, the sequence $\left\{x_{n}\right\}$ strongly converges to a point $p \in F$, where $p=Q_{F} f(p)$ and $Q_{F} f$ is a sunny nonexpansive retraction from $E$ onto $F$.

Proof. Step 1. We want to show that $\left\{x_{n}\right\}$ is bounded. Let $p \in F$. Then we have $p \in \operatorname{Fix}\left(S_{n}\right)$ for all $n \geq 1$ and $p \in A_{i}^{-1}(0)$ for all $i \geq 1$. Since each resolvent $J_{r_{n}}^{A_{i}}$ is a nonexpansive mapping for all $i \geq 1$ and $n \geq 1$, we obtain that

$$
\begin{aligned}
\left\|x_{n+1}-p\right\|=\left\|\alpha_{n, 0} f\left(x_{n}\right)+\sum_{i=1}^{\infty} \alpha_{n, i} S_{n} J_{r_{n}}^{A_{i}} x_{n}+e_{n}-p\right\| \\
\quad \leq\left\|\alpha_{n, 0} f\left(x_{n}\right)+\sum_{i=1}^{\infty} \alpha_{n, i} S_{n} J_{r_{n}}^{A_{i}} x_{n}-p\right\|+e_{n} \\
\leq \alpha_{n, 0}\left\|f\left(x_{n}\right)-p\right\|+\sum_{i=1}^{\infty} \alpha_{n, i}\left\|S_{n} J_{r_{n}}^{A_{i}} x_{n}-S_{n} p\right\|+e_{n} \\
\leq \alpha_{n, 0}\left(\left\|f\left(x_{n}\right)-f(p)\right\|+\|f(p)-p\|\right)+\sum_{i=1}^{\infty} \alpha_{n, i}\left\|J_{r_{n}}^{A_{i}} x_{n}-J_{r_{n}}^{A_{i}} p\right\|+e_{n} \\
\leq \alpha_{n, 0}\left(\left\|f\left(x_{n}\right)-f(p)\right\|+\|f(p)-p\|\right)+\sum_{i=1}^{\infty} \alpha_{n, i}\left\|x_{n}-p\right\|+e_{n} \\
\leq \alpha_{n, 0} k\left\|x_{n}-p\right\|+\left(1-\alpha_{n, 0}\right)\left\|x_{n}-p\right\|+\alpha_{n, 0}\|f(p)-p\|+e_{n} \\
=\left[\alpha_{n, 0} k+\left(1-\alpha_{n, 0}\right)\right]\left\|x_{n}-p\right\|+\alpha_{n, 0}\|f(p)-p\|+e_{n} \\
=\left[1-\alpha_{n, 0}(1-k)\right]\left\|x_{n}-p\right\|+\alpha_{n, 0}\|f(p)-p\|+e_{n} \\
=\left[1-\lambda_{n}\right]\left\|x_{n}-p\right\|+\lambda_{n} \frac{\|f(p)-p\|]}{(1-k)}+e_{n},
\end{aligned}
$$

where $\lambda_{n}:=\alpha_{n, 0}(1-k)$. Then, it follows that

$$
\begin{aligned}
\left\|x_{n+1}-p\right\| & \leq \max \left\{\left\|x_{n}-p\right\|, \frac{\|f(p)-p\|}{1-k}\right\}+e_{n} \\
& \leq \max \left\{\left\|x_{n-1}-p\right\|, \frac{\|f(p)-p\|}{1-k}\right\}+e_{n-1}+e_{n} \\
& \leq \ldots \leq \max \left\{\left\|x_{0}-p\right\|, \frac{\|f(p)-p\|}{1-k}\right\}+\sum_{n=1}^{\infty} e_{n} .
\end{aligned}
$$

This implies that the sequence $\left\{x_{n}\right\}$ is bounded. Also, we obtain that $\left\{f\left(x_{n}\right)\right\}$, $\left\{S_{n} J_{r_{n}}^{A_{i}} x_{n}\right\}$, and $\left\{J_{r_{n}}^{A_{i}} x_{n}\right\}$ are bounded for all $i \geq 1$ because $S_{n} J_{r_{n}}^{A_{i}}$ and $J_{r_{n}}^{A_{i}}$ are nonexpansive mappings for all $i \geq 1$ and $f$ is a contraction mapping. 
Step 2. We will show that $\lim _{n \rightarrow \infty}\left\|x_{n+1}-x_{n}\right\|=0$. This observes that

$$
\begin{aligned}
& \left\|x_{n+2}-x_{n+1}\right\|=\|\left(\alpha_{n+1,0} f\left(x_{n+1}\right)+\sum_{i=1}^{\infty} \alpha_{n+1, i} S_{n+1} J_{r_{n+1}}^{A_{i}} x_{n+1}+e_{n+1}\right) \\
& \quad-\left(\alpha_{n, 0} f\left(x_{n}\right)+\sum_{i=1}^{\infty} \alpha_{n, i} S_{n} J_{r_{n}}^{A_{i}} x_{n}+e_{n}\right) \| \\
& \leq\left\|\alpha_{n+1,0} f\left(x_{n+1}\right)-\alpha_{n, 0} f\left(x_{n}\right)-\alpha_{n+1,0} f\left(x_{n}\right)+\alpha_{n+1,0} f\left(x_{n}\right)\right\| \\
& +\left|e_{n+1}-e_{n}\right|+\| \sum_{i=1}^{\infty} \alpha_{n+1, i} S_{n+1} J_{r_{n+1}}^{A_{i}} x_{n+1}-\sum_{i=1}^{\infty} \alpha_{n+1, i} S_{n} J_{r_{n}}^{A_{i}} x_{n} \\
& \quad+\sum_{i=1}^{\infty} \alpha_{n+1, i} S_{n} J_{r_{n}}^{A_{i}} x_{n}-\sum_{i=1}^{\infty} \alpha_{n, i} S_{n} J_{r_{n}}^{A_{i}} x_{n} \| \\
& \alpha_{n+1,0}\left\|f\left(x_{n+1}\right)-f\left(x_{n}\right)\right\|+\left|\alpha_{n+1,0}-\alpha_{n+1,0}\right|\left\|f\left(x_{n}\right)\right\|+e_{n+1}+e_{n} \\
& \quad+\sum_{i=1}^{\infty} \alpha_{n+1, i}\left\|S_{n+1} J_{r_{n+1}}^{A_{i}} x_{n+1}-S_{n} J_{r_{n}}^{A_{i}} x_{n}\right\| \\
& \quad+\left\|\sum_{i=1}^{\infty} \alpha_{n+1, i} S_{n} J_{r_{n}}^{A_{i}} x_{n}-\sum_{i=1}^{\infty} \alpha_{n, i} S_{n} J_{r_{n}}^{A_{i}} x_{n}\right\| \\
& \alpha_{n+1,0} k\left\|x_{n+1}-x_{n}\right\|+\left|\alpha_{n+1,0}-\alpha_{n+1,0}\right|\left\|f\left(x_{n}\right)\right\|+e_{n+1}+e_{n} \\
& +\sum_{i=1}^{\infty} \alpha_{n+1, i}\left\|S_{n+1} J_{r_{n+1}}^{A_{i}} x_{n+1}-S_{n} J_{r_{n}}^{A_{i}} x_{n}\right\| \\
& +\left\|\sum_{i=1}^{\infty} \alpha_{n+1, i} S_{n} J_{r_{n}}^{A_{i}} x_{n}-\sum_{i=1}^{\infty} \alpha_{n, i} S_{n} J_{r_{n}}^{A_{i}} x_{n}\right\| .
\end{aligned}
$$

For each $i \geq 1$, by using Lemma 9 , we have the following estimate

$$
\begin{aligned}
& \left\|S_{n+1} J_{r_{n+1}}^{A_{i}} x_{n+1}-S_{n} J_{r_{n}}^{A_{i}} x_{n}\right\| \\
= & \left\|S_{n+1} J_{r_{n+1}}^{A_{i}} x_{n+1}-S_{n+1} J_{r_{n}}^{A_{i}} x_{n}+S_{n+1} J_{r_{n}}^{A_{i}} x_{n}-S_{n} J_{r_{n}}^{A_{i}} x_{n}\right\| \\
\leq & \left\|J_{r_{n+1}}^{A_{i}} x_{n+1}-J_{r_{n}}^{A_{i}} x_{n}\right\|+\left\|S_{n+1} J_{r_{n}}^{A_{i}} x_{n}-S_{n} J_{r_{n}}^{A_{i}} x_{n}\right\| \\
\leq & \left\|J_{r_{n+1}}^{A_{i}} x_{n+1}-J_{r_{n+1}}^{A_{i}} x_{n}+J_{r_{n}}^{A_{i}} x_{n}-J_{r_{n}}^{A_{i}} x_{n}\right\|+\left\|S_{n+1} J_{r_{n}}^{A_{i}} x_{n}-S_{n} J_{r_{n}}^{A_{i}} x_{n}\right\| \\
\leq & \left\|x_{n+1}-x_{n}\right\|+\left\|J_{r_{n}}^{A_{i}}\left(\frac{r_{n}}{r_{n+1}} x_{n}-\left(1-\frac{r_{n}}{r_{n+1}}\right) J_{r_{n+1}}^{A_{i}} x_{n}\right)-J_{r_{n}}^{A_{i}} x_{n}\right\| \\
& +\left\|S_{n+1} J_{r_{n}}^{A_{i}} x_{n}-S_{n} J_{r_{n}}^{A_{i}} x_{n}\right\| \\
\leq & \left\|x_{n+1}-x_{n}\right\|+\left|\frac{r_{n+1}-r_{n}}{r_{n+1}}\right|\left\|J_{r_{n+1}}^{A_{i}} x_{n}-x_{n}\right\|+\left\|S_{n+1} J_{r_{n}}^{A_{i}} x_{n}-S_{n} J_{r_{n}}^{A_{i}} x_{n}\right\| .
\end{aligned}
$$

Substituting it into the inequality (3.2), we obtain that

$$
\begin{aligned}
& \left\|x_{n+2}-x_{n+1}\right\| \leq \alpha_{n+1,0} k\left\|x_{n+1}-x_{n}\right\|+\left|\alpha_{n+1,0}-\alpha_{n+1,0}\right|\left\|f\left(x_{n}\right)\right\|+e_{n+1}+e_{n} \\
& \quad+\left\|\sum_{i=1}^{\infty} \alpha_{n+1, i} S_{n} J_{r_{n}}^{A_{i}} x_{n}-\sum_{i=1}^{\infty} \alpha_{n, i} S_{n} J_{r_{n}}^{A_{i}} x_{n}\right\|+\sum_{i=1}^{\infty} \alpha_{n+1, i}
\end{aligned}
$$




$$
\begin{aligned}
\{ & \left.\left\|x_{n+1}-x_{n}\right\|+\left|\frac{r_{n+1}-r_{n}}{r_{n+1}}\right|\left\|J_{r_{n+1}}^{A_{i}} x_{n}-x_{n}\right\|+\left\|S_{n+1} J_{r_{n}}^{A_{i}} x_{n}-S_{n} J_{r_{n}}^{A_{i}} x_{n}\right\|\right\} \\
= & \alpha_{n+1,0} k\left\|x_{n+1}-x_{n}\right\|+\left|\alpha_{n+1,0}-\alpha_{n+1,0}\right|\left\|f\left(x_{n}\right)\right\|+e_{n+1}+e_{n} \\
+ & \left\|\sum_{i=1}^{\infty} \alpha_{n+1, i} S_{n} J_{r_{n}}^{A_{i}} x_{n}-\sum_{i=1}^{\infty} \alpha_{n, i} S_{n} J_{r_{n}}^{A_{i}} x_{n}\right\|+\sum_{i=1}^{\infty} \alpha_{n+1, i}\left\|x_{n+1}-x_{n}\right\| \\
+ & \sum_{i=1}^{\infty} \alpha_{n+1, i}\left|\frac{r_{n+1}-r_{n}}{r_{n+1}}\right|\left\|J_{r_{n+1}}^{A_{i}} x_{n}-x_{n}\right\| \\
+ & \sum_{i=1}^{\infty} \alpha_{n+1, i}\left\|S_{n+1} J_{r_{n}}^{A_{i}} x_{n}-S_{n} J_{r_{n}}^{A_{i}} x_{n}\right\| \\
= & \left(1-\alpha_{n+1,0}(1-k)\right)\left\|x_{n+1}-x_{n}\right\|+\left|\alpha_{n+1,0}-\alpha_{n+1,0}\right|\left\|f\left(x_{n}\right)\right\|+e_{n+1}+e_{n} \\
+ & \left\|\sum_{i=1}^{\infty} \alpha_{n+1, i} S_{n} J_{r_{n}}^{A_{i}} x_{n}-\sum_{i=1}^{\infty} \alpha_{n, i} S_{n} J_{r_{n}}^{A_{i}} x_{n}\right\|+\sum_{i=1}^{\infty} \alpha_{n+1, i}\left|\frac{r_{n+1}-r_{n}}{r_{n+1}}\right| \\
& \times\left\|J_{r_{n+1}}^{A_{i}} x_{n}-x_{n}\right\|+\sum_{i=1}^{\infty} \alpha_{n+1, i}\left\|S_{n+1} J_{r_{n}}^{A_{i}} x_{n}-S_{n} J_{r_{n}}^{A_{i}} x_{n}\right\| \\
= & \left(1-\alpha_{n+1,0}(1-k)\right)\left\|x_{n+1}-x_{n}\right\|+c_{n},
\end{aligned}
$$

where

$$
\begin{aligned}
c_{n}:= & \left|\alpha_{n+1,0}-\alpha_{n+1,0}\right|\left\|f\left(x_{n}\right)\right\|+e_{n+1}+e_{n} \\
& +\left\|\sum_{i=1}^{\infty} \alpha_{n+1, i} S_{n} J_{r_{n}}^{A_{i}} x_{n}-\sum_{i=1}^{\infty} \alpha_{n, i} S_{n} J_{r_{n}}^{A_{i}} x_{n}\right\|+\sum_{i=1}^{\infty} \alpha_{n+1, i}\left|\frac{r_{n+1}-r_{n}}{r_{n+1}}\right| \\
& \times\left\|J_{r_{n+1}}^{A_{i}} x_{n}-x_{n}\right\|+\sum_{i=1}^{\infty} \alpha_{n+1, i}\left\|S_{n+1} J_{r_{n}}^{A_{i}} x_{n}-S_{n} J_{r_{n}}^{A_{i}} x_{n}\right\| .
\end{aligned}
$$

Since $\left\{x_{n}\right\},\left\{J_{r_{n}}^{A_{i}} x_{n}\right\}$ and $\left\{S_{n} J_{r_{n}}^{A_{i}} x_{n}\right\}$ are bounded sequences for all $i \geq 1$, then we can find the positive real numbers $L_{i}$ and $M_{i}$ such that

$$
\left\|S_{n} J_{r_{n}}^{A_{i}} x_{n}\right\| \leq L_{i} \leq \max _{i \geq 1}\left\{L_{i}\right\}:=L, \quad\left\|J_{r_{n}}^{A_{i}} x_{n}-x_{n}\right\| \leq M_{i} \leq \max _{i \geq 1}\left\{M_{i}\right\}:=M .
$$

Since $\lim _{n \rightarrow \infty} r_{n}=r>0$, thus $\lim _{n \rightarrow \infty} \frac{1}{r_{n}}$ exists and so there is a positive real number $r^{\prime}$ such that $\left|\frac{1}{r_{n}}\right|<r^{\prime}$ for all $n \in \mathbb{N}$. Then, we obtain that

$$
\begin{aligned}
\sum_{n=1}^{\infty}( & \left.\left\|\sum_{i=1}^{\infty} \alpha_{n+1, i} S_{n} J_{r_{n}}^{A_{i}} x_{n}-\sum_{i=1}^{\infty} \alpha_{n, i} S_{n} J_{r_{n}}^{A_{i}} x_{n}\right\|\right) \\
& \leq \sum_{n=1}^{\infty}\left(\left\|\sum_{i=1}^{\infty} \alpha_{n+1, i}-\sum_{i=1}^{\infty} \alpha_{n, i}\right\|\left\|S_{n} J_{r_{n}}^{A_{i}} x_{n}\right\|\right) \\
& \leq L \sum_{n=1}^{\infty}\left\|\sum_{i=1}^{\infty} \alpha_{n+1, i}-\sum_{i=1}^{\infty} \alpha_{n, i}\right\| \\
= & L \sum_{n=1}^{\infty}\left|\left(1-\alpha_{n+1,0}\right)-\left(1-\alpha_{n, 0}\right)\right|=L \sum_{n=1}^{\infty}\left|\alpha_{n+1,0}-\alpha_{n, 0}\right|<\infty
\end{aligned}
$$


and

$$
\begin{aligned}
& \sum_{n=1}^{\infty}\left(\sum_{i=1}^{\infty} \alpha_{n+1, i}\left|\frac{r_{n+1}-r_{n}}{r_{n+1}}\right|\left\|J_{r_{n+1}}^{A_{i}} x_{n}-x_{n}\right\|\right) \leq \sum_{n=1}^{\infty}\left(\sum_{i=1}^{\infty} \alpha_{n+1, i} r^{\prime} M\left|r_{n+1}-r_{n}\right|\right) \\
& \quad=r^{\prime} M \sum_{n=1}^{\infty}\left|r_{n+1}-r_{n}\right|\left(\sum_{i=1}^{\infty} \alpha_{n+1, i}\right)<\infty
\end{aligned}
$$

By the boundness of $\left\{J_{r_{n}}^{A_{i}} x_{n}\right\}$ and our assumptions, we observe that

$$
\begin{aligned}
& \sum_{n=1}^{\infty}\left(\sum_{i=1}^{\infty} \alpha_{n+1, i}\left\|S_{n+1} J_{r_{n}}^{A_{i}} x_{n}-S_{n} J_{r_{n}}^{A_{i}} x_{n}\right\|\right) \\
& \quad \leq \sum_{n=1}^{\infty}\left(\sum_{i=1}^{\infty} \alpha_{n+1, i} \sup \left\{\left\|S_{n+1} z-S_{n} z\right\|: z \in\left\{J_{r_{n}}^{A_{i}} x_{n}\right\}\right\}\right) \\
& \quad=\sum_{n=1}^{\infty} \sup \left\{\left\|S_{n+1} z-S_{n} z\right\|: z \in\left\{J_{r_{n}}^{A_{i}} x_{n}\right\}\right\}\left(\sum_{i=1}^{\infty} \alpha_{n+1, i}\right)<\infty .
\end{aligned}
$$

Since $\sum_{n=1}^{\infty}\left|\alpha_{n+1,0}-\alpha_{n, 0}\right|<\infty, \sum_{n=1}^{\infty} e_{n}<\infty$ and $\left\{f\left(x_{n}\right)\right\}$ is bounded, by (3.4), (3.5) and (3.6), then we see that the series $\sum_{n=1}^{\infty} c_{n}<\infty$. By using Lemma 12 and (3.3), we conclude that

$$
\lim _{n \rightarrow \infty}\left\|x_{n+1}-x_{n}\right\|=0
$$

Step 3. We will show that $\lim _{n \rightarrow \infty}\left\|x_{n}-J_{r}^{A_{i}} x_{n}\right\|=0$ for each $i \geq 1$ and $r>0$ where $r=\lim _{n \rightarrow \infty} r_{n}$. By using Lemma 5 and Lemma 8 , we obtain that

$$
\begin{aligned}
&\left\|x_{n+1}-p\right\|^{2}=\left\|\alpha_{n, 0} f\left(x_{n}\right)+\sum_{i=1}^{\infty} \alpha_{n, i} S_{n} J_{r_{n}}^{A_{i}} x_{n}+e_{n}-p\right\|^{2} \\
&=\left\|\left(\alpha_{n, 0} f\left(x_{n}\right)+\sum_{i=1}^{\infty} \alpha_{n, i} S_{n} J_{r_{n}}^{A_{i}} x_{n}-p\right)+e_{n}\right\|^{2} \\
&=\left\|\alpha_{n, 0} f\left(x_{n}\right)+\sum_{i=1}^{\infty} \alpha_{n, i} S_{n} J_{r_{n}}^{A_{i}} x_{n}-p\right\|^{2} \\
& \quad+2 e_{n}\left\|\alpha_{n, 0} f\left(x_{n}\right)+\sum_{i=1}^{\infty} \alpha_{n, i} S_{n} J_{r_{n}}^{A_{i}} x_{n}-p\right\|+e_{n}^{2} \\
&=\left\|\alpha_{n, 0} f\left(x_{n}\right)+\sum_{i=1}^{\infty} \alpha_{n, i} S_{n} J_{r_{n}}^{A_{i}} x_{n}-p\right\|^{2}+g_{n} \\
&=\left\|\alpha_{n, 0}\left(f\left(x_{n}\right)-p\right)+\sum_{i=1}^{\infty} \alpha_{n, i} S_{n} J_{r_{n}}^{A_{i}} x_{n}-\sum_{i=1}^{\infty} \alpha_{n, i} p\right\|^{2}+g_{n} \\
& \leq \alpha_{n, 0}\left\|f\left(x_{n}\right)-p\right\|^{2}+\sum_{i=1}^{\infty} \alpha_{n, i}\left\|J_{r_{n}}^{A_{i}} x_{n}-J_{r_{n}}^{A_{i}} p\right\|^{2}+g_{n}
\end{aligned}
$$




$$
\begin{aligned}
& \leq \alpha_{n, 0}\left\|f\left(x_{n}\right)-p\right\|^{2}+\sum_{i=1}^{\infty} \alpha_{n, i}\left\{\left\|x_{n}-p\right\|^{2}\right. \\
& \left.-2\left(\frac{\beta}{r_{n}}-K^{2}\right)\left\|\left(x_{n}-J_{r_{n}}^{A_{i}} x_{n}\right)-\left(p-J_{r_{n}}^{A_{i}} p\right)\right\|^{2}\right\}+g_{n} \\
& =\alpha_{n, 0}\left\|f\left(x_{n}\right)-p\right\|^{2}+\left(1-\alpha_{n, 0}\right)\left\|x_{n}-p\right\|^{2} \\
& -\sum_{i=1}^{\infty} 2 \alpha_{n, i}\left(\frac{\beta}{r_{n}}-K^{2}\right)\left\|\left(x_{n}-J_{r_{n}}^{A_{i}} x_{n}\right)\right\|^{2}+g_{n},
\end{aligned}
$$

where $g_{n}=2 e_{n}\left\|\alpha_{n, 0} f\left(x_{n}\right)+\sum_{i=1}^{\infty} \alpha_{n, i} S_{n} J_{r_{n}}^{A_{i}} x_{n}-p\right\|+e_{n}^{2}$. Since $\left\{f\left(x_{n}\right)\right\}$ and $\left\{S_{n} J_{r_{n}}^{A_{i}} x_{n}\right\}$ are bounded sequences, $\sum_{i=1}^{\infty} \alpha_{n, i}<1$ and $\lim _{n \rightarrow \infty} e_{n}=0$, we have $\lim _{n \rightarrow \infty} g_{n}=0$. From (3.7), we get that

$$
\begin{aligned}
& \sum_{i=1}^{\infty} 2 \alpha_{n, i}\left(\frac{\beta}{r_{n}}-K^{2}\right)\left\|\left(x_{n}-J_{r_{n}}^{A_{i}} x_{n}\right)\right\|^{2} \\
\leq & \alpha_{n, 0}\left\|f\left(x_{n}\right)-p\right\|^{2}+\left(1-\alpha_{n, 0}\right)\left\|x_{n}-p\right\|^{2}-\left\|x_{n+1}-p\right\|^{2}+g_{n} \\
\leq & \left(1-\alpha_{n, 0}\right)\left\|x_{n}-p\right\|^{2}-\left\|x_{n+1}-p\right\|^{2}+\alpha_{n, 0}\left\|f\left(x_{n}\right)-p\right\|^{2}+g_{n} \\
\leq & \left\|x_{n}-x_{n+1}\right\|\left\|x_{n}-p+x_{n+1}-p\right\|+\alpha_{n, 0}\left\|f\left(x_{n}\right)-p\right\|^{2}+g_{n} .
\end{aligned}
$$

Let us fix $i \in \mathbb{N}$. Since $\alpha_{n, i} \subset(0,1)$ for all $i \in \mathbb{N}$ and $2\left(\frac{\beta}{r_{n}}-K^{2}\right)>0$. Then, by $(3.8)$, we get that

$$
\begin{gathered}
2 \alpha_{n, i}\left(\frac{\beta}{r_{n}}-K^{2}\right)\left\|x_{n}-J_{r_{n}}^{A_{i}} x_{n}\right\|^{2} \leq \sum_{i=1}^{\infty} 2 \alpha_{n, i}\left(\frac{\beta}{r_{n}}-K^{2}\right)\left\|\left(x_{n}-J_{r_{n}}^{A_{i}} x_{n}\right)\right\|^{2} \\
\leq\left\|x_{n}-x_{n+1}\right\|\left\|x_{n}-p+x_{n+1}-p\right\|+\alpha_{n, 0}\left\|f\left(x_{n}\right)-p\right\|^{2}+g_{n} .
\end{gathered}
$$

Since $\left\{x_{n}\right\}$ and $\left\{f\left(x_{n}\right)\right\}$ are bounded sequences, $\lim _{n \rightarrow \infty} g_{n}=0, \lim _{n \rightarrow \infty} \alpha_{n, 0}$, $\lim _{n \rightarrow \infty} \alpha_{n, i}=\alpha_{i}$ for each $i \geq 1$ and by (3.9), we conclude that

$$
\lim _{n \rightarrow \infty}\left\|x_{n}-J_{r_{n}}^{A_{i}} x_{n}\right\|=0 .
$$

By using Lemma 9 we note that

$$
\begin{aligned}
\left\|x_{n}-J_{r}^{A_{i}} x_{n}\right\| & \leq\left\|x_{n}-J_{r_{n}}^{A_{i}} x_{n}\right\|+\left\|J_{r_{n}}^{A_{i}} x_{n}-J_{r}^{A_{i}} x_{n}\right\| \\
& =\left\|x_{n}-J_{r_{n}}^{A_{i}} x_{n}\right\|+\left\|J_{r}^{A_{i}}\left(\frac{r}{r_{n}} x_{n}+\left(1-\frac{r}{r_{n}}\right) J_{r_{n}}^{A_{i}} x_{n}\right)-J_{r}^{A_{i}} x_{n}\right\| \\
& \leq\left\|x_{n}-J_{r_{n}}^{A_{i}} x_{n}\right\|+\left\|\left(\frac{r}{r_{n}} x_{n}-\left(1-\frac{r}{r_{n}}\right) J_{r_{n}}^{A_{i}} x_{n}\right)-x_{n}\right\| \\
& =\left\|x_{n}-J_{r_{n}}^{A_{i}} x_{n}\right\|+\mid 1-\frac{r}{r_{n}}\left\|J_{r_{n}}^{A_{i}} x_{n}-x_{n}\right\| \\
& \leq\left\|x_{n}-J_{r_{n}}^{A_{i}} x_{n}\right\|+\left\|J_{r_{n}}^{A_{i}} x_{n}-x_{n}\right\|=2\left\|x_{n}-J_{r_{n}}^{A_{i}} x_{n}\right\| .
\end{aligned}
$$

Hence, by (3.10), we observe that

$$
\lim _{n \rightarrow \infty}\left\|x_{n}-J_{r}^{A_{i}} x_{n}\right\|=0 .
$$


Step 4. We will show that there exists a unique point $p \in F$ such that

$$
\limsup _{n \rightarrow \infty}\left\langle f(p)-p, j\left(x_{n}-p\right)\right\rangle \leq 0, \quad p=Q_{F} f(p) .
$$

Since $\left\{x_{n}\right\}$ is bounded, there exists a subsequence $\left\{x_{n_{m}}\right\}$ of $\left\{x_{n}\right\}$ which converges weakly to $\bar{x}$. To prove this, we divide to 4 steps.

Step 4.1. We will show that $\bar{x} \in \bigcap_{i=1}^{\infty} A_{i}^{-1}(0) \neq \emptyset$. Note that

$$
\begin{aligned}
\left\|x_{n_{m}}-J_{r}^{A_{i}} \bar{x}\right\| & \leq\left\|x_{n_{m}}-J_{r}^{A_{i}} x_{n_{m}}\right\|+\left\|J_{r}^{A_{i}} x_{n_{m}}-J_{r}^{A_{i}} \bar{x}\right\| \\
& \leq\left\|x_{n_{m}}-J_{r}^{A_{i}} x_{n_{m}}\right\|+\left\|x_{n_{m}}-\bar{x}\right\| .
\end{aligned}
$$

This implies that

$$
\limsup _{m \rightarrow \infty}\left\|x_{n_{m}}-J_{r}^{A_{i}} \bar{x}\right\| \leq \limsup _{m \rightarrow \infty}\left\|x_{n_{m}}-\bar{x}\right\| .
$$

Since a smooth Banach space with a weakly sequential continuous duality mapping $j$ has the Opial's property. Then, by using (3.13), we obtain that $J_{r}^{A_{i}} \bar{x}=\bar{x}$ for all $i \in \mathbb{N}$. This is complete the proof because

$$
\begin{aligned}
J_{r}^{A_{i}} \bar{x}=\bar{x}, \quad \forall i \in \mathbb{N}, & \Longleftrightarrow \bar{x} \in F i x\left(J_{r}^{A_{i}}\right), \quad \forall i \in \mathbb{N} \\
& \Longleftrightarrow \bar{x} \in A_{i}^{-1}(0), \quad \forall i \in \mathbb{N} \\
& \Longleftrightarrow \bar{x} \in \bigcap_{i=1}^{\infty} A_{i}^{-1}(0) .
\end{aligned}
$$

Step 4.2. Next, we will show that $\bar{x} \in \operatorname{Fix}(S)=\bigcap_{n=1}^{\infty} \operatorname{Fix}\left(S_{n}\right)$. Note that

$$
\left\|S_{n} J_{r_{n}}^{A_{i}} x_{n}-J_{r_{n}}^{A_{i}} x_{n}\right\| \leq\left\|S_{n} J_{r_{n}}^{A_{i}} x_{n}-x_{n+1}\right\|+\left\|x_{n+1}-x_{n}\right\|+\left\|x_{n}-J_{r_{n}}^{A_{i}} x_{n}\right\| .
$$

We observe that

$$
\begin{aligned}
& \left\|x_{n+1}-S_{n} J_{r_{n}}^{A_{i}} x_{n}\right\|=\left\|\alpha_{n, 0} f\left(x_{n}\right)+\sum_{i=1}^{\infty} \alpha_{n, i} S_{n} J_{r_{n}}^{A_{i}} x_{n}+e_{n}-S_{n} J_{r_{n}}^{A_{i}} x_{n}\right\| \\
& \leq\left\|\alpha_{n, 0} f\left(x_{n}\right)+\sum_{i=1}^{\infty} \alpha_{n, i} S_{n} J_{r_{n}}^{A_{i}} x_{n}-S_{n} J_{r_{n}}^{A_{i}} x_{n}\right\|+e_{n} \\
& =\left\|\alpha_{n, 0} f\left(x_{n}\right)+\sum_{i=1}^{\infty} \alpha_{n, i} S_{n} J_{r_{n}}^{A_{i}} x_{n}-\left(\sum_{i=0}^{\infty} \alpha_{n, i}\right) S_{n} J_{r_{n}}^{A_{i}} x_{n}\right\|+e_{n} \\
& =\left\|\alpha_{n, 0} f\left(x_{n}\right)+\sum_{i=1}^{\infty} \alpha_{n, i} S_{n} J_{r_{n}}^{A_{i}} x_{n}-\left(\alpha_{n, 0}+\sum_{i=1}^{\infty} \alpha_{n, i}\right) S_{n} J_{r_{n}}^{A_{i}} x_{n}\right\|+e_{n} \\
& \leq \alpha_{n, 0}\left\|f\left(x_{n}\right)-S_{n} J_{r_{n}}^{A_{i}} x_{n}\right\|+e_{n} .
\end{aligned}
$$

Since $\left\{f\left(x_{n}\right)\right\}$ and $\left\{S_{n} J_{r_{n}}^{A_{i}} x_{n}\right\}$ are bounded sequences, by condition (a) and (d), we obtain that

$$
\lim _{n \rightarrow \infty}\left\|S_{n} J_{r_{n}}^{A_{i}} x_{n}-x_{n}\right\|=0
$$


Note that

$$
\begin{aligned}
\left\|S x_{n}-x_{n}\right\| & \leq\left\|S x_{n}-S J_{r_{n}}^{A_{i}} x_{n}\right\|+\left\|S J_{r_{n}}^{A_{i}} x_{n}-S_{n} J_{r_{n}}^{A_{i}} x_{n}\right\|+\left\|S_{n} J_{r_{n}}^{A_{i}} x_{n}-x_{n}\right\| \\
& \leq\left\|x_{n}-J_{r_{n}}^{A_{i}} x_{n}\right\|+\left\|S J_{r_{n}}^{A_{i}} x_{n}-S_{n} J_{r_{n}}^{A_{i}} x_{n}\right\|+\left\|S_{n} J_{r_{n}}^{A_{i}} x_{n}-x_{n}\right\| .
\end{aligned}
$$

By using Lemma 11, (3.12) and (3.14), we obtain that

$$
\lim _{n \rightarrow \infty}\left\|S x_{n}-x_{n}\right\|=0
$$

Since $x_{n_{m}} \rightarrow \bar{x}$, by Lemma 10, we have $\bar{x} \in \operatorname{Fix}(S)=\bigcap_{n=1}^{\infty} \operatorname{Fix}\left(S_{n}\right)$.

Step 4.3. We will show that there exists a unique point $p \in F$ and $p=$ $Q_{F} f(p)$. Indeed, since $F:=\left(\bigcap_{n=1}^{\infty} F i x\left(S_{n}\right)\right) \bigcap\left(\bigcap_{i=1}^{\infty} A_{i}^{-1}(0)\right) \neq \emptyset$ is closed and convex. By using Lemma 2, we obtain that the set $F$ is a sunny nonexpansive retract from $E$ onto $F$, i.e., $Q_{F}$ is well defined. We see that $Q_{F} f$ is contraction of $C$ into itself. In fact, since $Q_{F}$ is a nonexpansive mapping,

$$
\left\|Q_{F}(f)(x)-Q_{F}(f)(y)\right\| \leq\|f(x)-f(y)\| \leq k\|x-y\|
$$

where $k \in(0,1)$. By Banach contraction principle, there exists a unique element $p \in F$ such that $p=Q_{F} f(p)$. That is $\bar{x}=p=Q_{F} f(p) \in F$.

Step 4.4. We will show that $\limsup _{n \rightarrow \infty}\left\langle f(p)-p, j\left(x_{n}-p\right)\right\rangle \leq 0$.

Choose a subsequence $\left\{x_{n_{m}}\right\}$ of $\left\{x_{n}\right\}$ such that

$$
\limsup _{n \rightarrow \infty}\left\langle f(p)-p, j\left(x_{n}-p\right)\right\rangle=\lim _{n \rightarrow \infty}\left\langle f(p)-p, j\left(x_{n_{m}}-p\right)\right\rangle .
$$

Since $\left\{x_{n}\right\}$ is bounded, there exists a subsequence $\left\{x_{n_{m_{j}}}\right\}$ of $\left\{x_{n_{m}}\right\}$ which $x_{n_{m_{j}}} \rightarrow \bar{x}$. Without loss of generality, we can assume that $x_{n_{m}} \rightarrow \bar{x}$. It follows that

$$
\begin{aligned}
\limsup _{n \rightarrow \infty}\left\langle f(p)-p, j\left(x_{n}-p\right)\right\rangle & =\lim _{m \rightarrow \infty}\left\langle f(p)-p, j\left(x_{n_{m}}-p\right)\right\rangle \\
& =\langle f(p)-p, j(\bar{x}-p)\rangle \leq 0
\end{aligned}
$$

Step 5. Finally, we will show that $x_{n} \rightarrow p$.

By using Lemma 7 and Lemma 5, we have

$$
\begin{aligned}
& \left\|x_{n+1}-p\right\|^{2}=\left\|\alpha_{n, 0} f\left(x_{n}\right)+\sum_{i=1}^{\infty} \alpha_{n, i} S_{n} J_{r_{n}}^{A_{i}} x_{n}+e_{n}-p\right\|^{2} \\
& \leq\left\|\sum_{i=1}^{\infty} \alpha_{n, i} S_{n} J_{r_{n}}^{A_{i}} x_{n}-\sum_{i=1}^{\infty} \alpha_{n, i} p+e_{n}\right\|^{2}+2 \alpha_{n, 0}\left\langle f\left(x_{n}\right)-p, j\left(x_{n+1}-p\right)\right\rangle \\
& \leq\left\|\sum_{i=1}^{\infty} \alpha_{n, i} S_{n} J_{r_{n}}^{A_{i}} x_{n}-\sum_{i=1}^{\infty} \alpha_{n, i} p\right\|^{2}+2 \alpha_{n, 0}\left\langle f\left(x_{n}\right)-p, j\left(x_{n+1}-p\right)\right\rangle \\
& \quad+2 e_{n}\left\|\sum_{i=1}^{\infty} \alpha_{n, i} S_{n} J_{r_{n}}^{A_{i}} x_{n}-\sum_{i=1}^{\infty} \alpha_{n, i} p\right\|+e_{n}^{2}
\end{aligned}
$$




$$
\begin{aligned}
\leq \quad & \left\|\sum_{i=1}^{\infty} \alpha_{n, i}\left(S_{n} J_{r_{n}}^{A_{i}} x_{n}-p\right)\right\|^{2}+h_{n}+2 \alpha_{n, 0}\left\langle f\left(x_{n}\right)-p, j\left(x_{n+1}-p\right)\right\rangle \\
\leq \quad & \left(1-\alpha_{n, 0}\right)^{2}\left\|x_{n}-p\right\|^{2}+h_{n}+2 \alpha_{n, 0}\left\langle f\left(x_{n}\right)-f(p), j\left(x_{n+1}-p\right)\right\rangle \\
& +2 \alpha_{n, 0}\left\langle f(p)-p, j\left(x_{n+1}-p\right)\right\rangle \\
\leq \quad & \left(1-\alpha_{n, 0}\right)^{2}\left\|x_{n}-p\right\|^{2}+h_{n}+2 \alpha_{n, 0}\left\|f\left(x_{n}\right)-f(p)\right\|\left\|x_{n+1}-p\right\| \\
& +2 \alpha_{n, 0}\left\langle f(p)-p, j\left(x_{n+1}-p\right)\right\rangle \\
\leq \quad & \left(1-\alpha_{n, 0}\right)^{2}\left\|x_{n}-p\right\|^{2}+h_{n}+2 \alpha_{n, 0} k\left\|x_{n}-p\right\|\left\|x_{n+1}-p\right\| \\
& +2 \alpha_{n, 0}\left\langle f(p)-p, j\left(x_{n+1}-p\right)\right\rangle \\
\leq \quad & \left(1-\alpha_{n, 0}\right)^{2}\left\|x_{n}-p\right\|^{2}+h_{n}+\alpha_{n, 0} k\left\{\left\|x_{n}-p\right\|^{2}+\left\|x_{n+1}-p\right\|^{2}\right\} \\
& +2 \alpha_{n, 0}\left\langle f(p)-p, j\left(x_{n+1}-p\right)\right\rangle,
\end{aligned}
$$

where $h_{n}=2 e_{n}\left\|\sum_{i=1}^{\infty} \alpha_{n, i} S_{n} J_{r_{n}}^{A_{i}} x_{n}-\sum_{i=1}^{\infty} \alpha_{n, i} p\right\|+e_{n}^{2}$. This implies that

$$
\begin{aligned}
\left\|x_{n+1}-p\right\|^{2} \leq & \frac{\left(1-\alpha_{n, 0}\right)^{2}+\alpha_{n, 0} k}{1-\alpha_{n, 0} k}\left\|x_{n}-p\right\|^{2} \\
& +\frac{2 \alpha_{n, 0}}{1-\alpha_{n, 0} k}\left\langle f(p)-p, j\left(x_{n+1}-p\right)\right\rangle+\frac{h_{n}}{\left(1-\alpha_{n, 0} k\right)} \\
= & \frac{1-2 \alpha_{n, 0}+\alpha_{n, 0} k}{1-\alpha_{n, 0} k}\left\|x_{n}-p\right\|^{2}+\frac{\alpha_{n, 0}^{2}}{1-\alpha_{n, 0} k}\left\|x_{n}-p\right\|^{2} \\
& +\frac{2 \alpha_{n, 0}}{1-\alpha_{n, 0} k}\left\langle f(p)-p, j\left(x_{n+1}-p\right)\right\rangle+\frac{h_{n}}{\left(1-\alpha_{n, 0} k\right)} \\
\leq & \left(1-\frac{2(1-k) \alpha_{n, 0}}{1-\alpha_{n, 0} k}\right)\left\|x_{n}-p\right\|^{2}+\frac{2(1-k) \alpha_{n, 0}}{1-\alpha_{n, 0} k}\left(\frac{\alpha_{n, 0} M}{2(1-k)}\right. \\
& \left.+\frac{1}{1-k}\left\langle f(p)-p, j\left(x_{n+1}-p\right)\right\rangle\right)+\frac{h_{n}}{\left(1-\alpha_{n, 0} k\right)} \\
\leq & \left(1-t_{n}\right)\left\|x_{n}-p\right\|^{2}+t_{n} b_{n}+c_{n}^{\prime},
\end{aligned}
$$

where $\tilde{M}=\sup \left\{\left\|x_{n}-p\right\|^{2}: n \geq 0\right\}, t_{n}=\frac{2(1-k) \alpha_{n, 0}}{1-\alpha_{n, 0} k}, b_{n}=\frac{\alpha_{n, 0} \tilde{M}}{2(1-k)}+\frac{1}{1-k}\langle f(p)-$ $p, j\left(x_{n+1}-p\right\rangle$, and $c_{n}^{\prime}=\frac{h_{n}}{\left(1-\alpha_{n, 0}\right)}$. By assumptions, we see that $\lim _{n \rightarrow \infty} t_{n}=0$, $\sum_{n=1}^{\infty} t_{n}=\infty$ and $\sum_{n=1}^{\infty} c_{n}^{\prime}<\infty$. By step 4.4, so we have $\limsup _{n \rightarrow \infty} b_{n} \leq 0$. Therefore, by using Lemma 12 , we obtain that the sequence $\left\{x_{n}\right\}$ strongly converges to $p=Q_{F} f(p)$. This completes the proof.

Remark 2. We given some examples concerning of the sequence $\left\{\alpha_{n, 0}\right\}$ and $\left\{\alpha_{n, i}\right\}$ that satisfy the control condition of Theorem 1 as follows:

(1) if $\alpha_{n, 0}:=\frac{1}{n}$ and $\alpha_{n, i}:=\left(1-\frac{1}{n}\right) \frac{1}{2^{i}}, n \geq 1$.

It easy to see that

$$
\lim _{n \rightarrow \infty} \alpha_{n, 0}=\lim _{n \rightarrow \infty} \frac{1}{n}=0, \quad \sum_{n=1}^{\infty} \alpha_{n, 0}=\sum_{n=1}^{\infty} \frac{1}{n}=\infty,
$$




$$
\sum_{n=1}^{\infty}\left|\alpha_{n+1,0}-\alpha_{n, 0}\right|=\sum_{n=1}^{\infty}\left|\frac{1}{n+1}-\frac{1}{n}\right| \leq \sum_{n=1}^{\infty} \frac{1}{n^{2}}<\infty .
$$

For each $i \geq 1$, we have

$$
\lim _{n \rightarrow \infty} \alpha_{n, i}=\lim _{n \rightarrow \infty}\left(1-\frac{1}{n}\right) \frac{1}{2^{i}}=\frac{1}{2^{i}} \in(0,1) .
$$

Since $\sum_{i=1}^{\infty} \frac{1}{2^{i}}=1$, then

$$
\begin{aligned}
\sum_{i=0}^{\infty} \alpha_{n, i} & =\alpha_{n, 0}+\sum_{i=1}^{\infty} \alpha_{n, i}=\frac{1}{n}+\sum_{i=1}^{\infty}\left(1-\frac{1}{n}\right) \frac{1}{2^{i}} \\
& =\frac{1}{n}+\left(1-\frac{1}{n}\right) \sum_{i=1}^{\infty} \frac{1}{2^{i}}=1 .
\end{aligned}
$$

(2) if $\alpha_{n, 0}:=\frac{1}{n+1}$ and $\alpha_{n, i}:=\left(1-\frac{1}{n+1}\right) \frac{1}{2^{i}}, n \geq 1$.

(3) if $\alpha_{n, 0}:=\frac{1}{\sqrt{n}}$ and $\alpha_{n, i}:=\left(1-\frac{1}{\sqrt{n}}\right) \frac{1}{2^{i}}, n \geq 1, i \geq 1$.

(4) if $\alpha_{n, 0}:=\frac{1}{n^{p}}$ and $\alpha_{n, i}:=\left(1-\frac{1}{n^{p}}\right) \frac{1}{2^{i}}, n \geq 1, i \geq 1, p \leq 1$.

By setting $S_{n} \equiv I$ for all $n \in \mathbb{N}$ in Theorem 1 , we obtain the following Corollary:

Corollary 1. Let $E$ be a uniformly convex and 2-uniformly smooth of real Banach space and let $C$ be a nonempty closed convex subset of $E$ which has a weakly continuous duality mapping $J$ from $E$ to $E^{*}$. For any $i \in \mathbb{N}$, let $\left\{A_{i}\right\}_{i=1}^{\infty}$ be an infinite sequence of $\beta_{i}$-inverse strongly accretive operators in $E$ such that $\beta:=\inf _{i \geq 1}\left\{\beta_{i}\right\}>0$ and $\bigcap_{i=1}^{\infty} \overline{D\left(A_{i}\right)} \subset C \subset \bigcap_{i=1}^{\infty} R\left(I+r A_{i}\right)$ for all $r>0$. Let $f: C \rightarrow C$ be a contraction mapping with a constant $k \in(0,1)$ and $J_{r_{n}}^{A_{i}}=\left(I+r_{n} A_{i}\right)^{-1}$ be a resolvent of $A_{i}$ for $r_{n}>0$. For given $x_{1} \in C$, let $\left\{x_{n}\right\}$ be a sequence defined by the following:

$$
x_{n+1}=\alpha_{n, 0} f\left(x_{n}\right)+\sum_{i=1}^{\infty} \alpha_{n, i} J_{r_{n}}^{A_{i}} x_{n}+e_{n}, \quad \forall n \in \mathbb{N},
$$

where $\sum_{i=0}^{\infty} \alpha_{n, i}=1,\left\{\alpha_{i}\right\} \subset(0,1)$ for all $i \geq 0,\left\{r_{n}\right\} \subset\left(0, \frac{\beta}{K^{2}}\right)$ where $K$ is the 2-uniformly smooth constant, and $\left\{e_{n}\right\} \subset(0, \infty)$. Suppose that the following conditions hold:

(a) $\lim _{n \rightarrow \infty} \alpha_{n, 0}=0, \sum_{n=1}^{\infty} \alpha_{n, 0}=\infty$ and $\sum_{n=1}^{\infty}\left|\alpha_{n+1,0}-\alpha_{n, 0}\right|<\infty$;

(b) for each $i \geq 1, \lim _{n \rightarrow \infty} \alpha_{n, i}=\alpha_{i} \in(0,1)$;

(c) $\sum_{n=1}^{\infty}\left|r_{n+1}-r_{n}\right|<\infty$ and $\lim _{n \rightarrow \infty} r_{n}=r>0$;

(d) $\sum_{n=1}^{\infty} e_{n}<\infty$. 
Then, the sequence $\left\{x_{n}\right\}$ strongly converges to a point $p \in F$, where $p=$ $Q_{F} f(x)$.

If we put $J_{r_{n}}^{A_{i}} \equiv I$ for all $i \in \mathbb{N}, n \in \mathbb{N}$ in Theorem 1 , we obtain the following Corollary:

Corollary 2. Let $E$ be a uniformly convex and 2-uniformly smooth of real Banach space and let $C$ be a nonempty closed convex subset of $E$ which has a weakly continuous duality mapping $j$ from $E$ to $E^{*}$. For any $n \in \mathbb{N}$, let $\left\{S_{n}\right\}_{n=1}^{\infty}$ be an infinite sequence of nonexpansive mapping from $C$ onto itself such that $F:=\bigcap_{n=1}^{\infty} \operatorname{Fix}\left(S_{n}\right) \neq \emptyset$. Let $f: C \rightarrow C$ be a contraction mapping with a constant $k \in(0,1)$. For given $x_{1} \in C$, let $\left\{x_{n}\right\}$ be a sequence defined by the following:

$$
x_{n+1}=\alpha_{n} f\left(x_{n}\right)+\left(1-\alpha_{n}\right) S_{n} x_{n}+e_{n}, \quad \forall n \geq 1 .
$$

If sequence $\left\{\alpha_{n}\right\} \subset(0,1)$ and $\left\{e_{n}\right\} \subset(0, \infty)$ are satisfy the following conditions:

(a) $\lim _{n \rightarrow \infty} \alpha_{n}=0, \sum_{n=1}^{\infty} \alpha_{n}=\infty$ and $\sum_{n=1}^{\infty}\left|\alpha_{n+1}-\alpha_{n}\right|<\infty$;

(b) $\sum_{n=1}^{\infty} e_{n}<\infty$.

Assume that $\sum_{n=1}^{\infty} \sup \left\{\left\|S_{n+1} x-S_{n} x\right\|: x \in B\right\}<\infty$ for any bounded subset $B$ of $C$. Let $S$ be a mapping of $C$ into itself defined by $S x=\lim _{n \rightarrow \infty} S_{n} x$ for all $x \in C$ and suppose that $\operatorname{Fix}(S)=\bigcap_{n=1}^{\infty} \operatorname{Fix}\left(S_{n}\right)$. Then, the sequence $\left\{x_{n}\right\}$ converges strongly to a point $p \in F$, where $p=Q_{F} f(x)$ and $Q_{F} f(x)$ is sunny nonexpansive retraction from $E$ onto $F$.

It is well known that in a Hilbert space, the 2-uniformly smooth constant $K=1$. If we set $E=H$ in Theorem 1, we obtain the following Corollary:

Corollary 3. Let $C$ be a nonempty close convex subset of a Hilbert space $H$. For any $i \in \mathbb{N}$, let $\left\{A_{i}\right\}_{i=1}^{\infty}$ be an infinite sequence of $\beta_{i}$-inverse strongly accretive operators in $H$ such that $\beta:=\inf _{i \geq 1}\left\{\beta_{i}\right\}>0$ and $\bigcap_{i=1}^{\infty} \overline{D\left(A_{i}\right)} \subseteq C \subseteq$ $\bigcap_{i=1}^{\infty} R\left(I+r A_{i}\right)$ for all $r>0$. Let $\left\{S_{n}\right\}_{n=1}^{\infty}$ be an infinite sequence of nonexpansive mapping from $C$ onto itself such that $F:=\bigcap_{n=1}^{\infty} F i x\left(S_{n}\right) \bigcap \bigcap_{i=1}^{\infty} A_{i}^{-1}(0) \neq$ $\emptyset$. Let $f: C \rightarrow C$ be a contraction mapping with a constant $k \in(0,1)$ and $J_{r_{n}}^{A_{i}}=\left(I+r_{n} A_{i}\right)^{-1}$ be a resolvent of $A_{i}$ for $r_{n}>0$. For given $x_{1} \in \bigcap_{i=1}^{\infty} \overline{D\left(A_{i}\right)}$, let $\left\{x_{n}\right\}$ be a sequence defined by the following:

$$
x_{n+1}=\alpha_{n, 0} f\left(x_{n}\right)+\sum_{i=1}^{\infty} \alpha_{n, i} S_{n} J_{r_{n}}^{A_{i}} x_{n}+e_{n}, \quad \forall n \geq 1,
$$

where $\sum_{i=0}^{\infty} \alpha_{n, i}=1$ and sequence $\left\{\alpha_{n, i}\right\} \subset(0,1)$ for $i \geq 0$. If sequence $\left\{r_{n}\right\} \subset(0, \beta)$, and $\left\{e_{n}\right\} \subset(0, \infty)$ are satisfy the following conditions:

(a) $\lim _{n \rightarrow \infty} \alpha_{n, 0}=0, \sum_{n=1}^{\infty} \alpha_{n, 0}=\infty$ and $\sum_{n=1}^{\infty}\left|\alpha_{n+1,0}-\alpha_{n, 0}\right|<\infty$;

(b) for each $i \geq 1, \lim _{n \rightarrow \infty} \alpha_{n, i}=\alpha_{i} \in(0,1)$;

(c) $\sum_{n=1}^{\infty}\left|r_{n+1}-r_{n}\right|<\infty$ and $\lim _{n \rightarrow \infty} r_{n}=r>0$; 
(d) $\sum_{n=1}^{\infty} e_{n}<\infty$.

Assume that $\sum_{n=1}^{\infty} \sup \left\{\left\|S_{n+1} x-S_{n} x\right\|: x \in B\right\}<\infty$ for any bounded subset $B$ of $C$. Let $S$ be a mapping of $C$ into itself defined by $S x=\lim _{n \rightarrow \infty} S_{n} x$ for all $x \in C$ and suppose that $F i x(S)=\bigcap_{n=1}^{\infty} F i x\left(S_{n}\right)$. Then, the sequence $\left\{x_{n}\right\}$ strongly converges to a point $p \in F$ and $p=P_{F} f(x)$ and $P_{C} f(x)$ is a metric projection from $H$ onto $F$.

Remark 3. We note that Theorem 1 improves and extends in the following aspects:

(a) Our result can be extend [8, Theorem 3.1] from a Hilbert space to a uniformly convex and 2-uniformly smooth Banach space.

(b) Our result can be extend from solving the problems of finding a common element of a finite sequence of monotone operators [8, Theorem 3.1] to solving the problems of finding a common element of an infinite family of inverse-strongly accretive operators.

(c) The iterative scheme (3.1) in Theorem 1 is different from Theorem 3.1 in [8] because the $J_{r_{n}}^{A_{i}}$ is replace by the composite mapping $S_{n} J_{r_{n}}^{A_{i}}$.

\section{Numerical examples}

Let us show numerical example to demonstrate the performance and convergence of our main result as follows.

Example 1. Let $E=\mathbb{R}$ and $C=[-1,000,1,000]$. Define an infinite sequence of mappings $S_{n}: C \rightarrow C$ and operators $A_{i}: \mathbb{R} \rightarrow \mathbb{R}$ by

$$
S_{n}(x)=\frac{x-1}{n+1}+1 \text { for all } n \in \mathbb{N}
$$

and

$$
A_{i}(x)=\frac{i}{i+1}(x-1) \text { for all } i \in \mathbb{N} \text {. }
$$

It is not hard to see that $S_{n}$ are nonexpansive mappings for all $n \in \mathbb{N}$ such that

$$
\sum_{n=1}^{\infty} \sup \left\{\left\|S_{n+1} x-S_{n} x\right\|: x \in B\right\}<\infty
$$

for any bounded subset $B$ of $C$ and $A_{i}$ are $\beta_{i}$-inverse strongly accretive operators where $\beta_{i}=1+\frac{1}{i}$ for all $i \in \mathbb{N}$. So $\beta:=\inf _{i \geq 1}\left\{\beta_{i}\right\}=1>0$. Moreover, we see that the resolvent of each $A_{i}$ is

$$
J_{r_{n}}^{A_{i}}(x)=\left(I+r_{n} A_{i}\right)^{-1}(x)=\frac{(1+i) x+r_{n} i}{1+i+r_{n} i} \text { for } r_{n}>0 .
$$

Note that the resolvent $J_{r_{n}}^{A_{i}}$ are nonexpansive mappings for fixed $i \in \mathbb{N}$. 
By the definition of $S_{n}$ and $A_{i}$, we observe that $1 \in F$. The Theorem 1 certifies that the sequence $x_{n}$ in Algorithm 1 converses to the point in $F$. That is $x_{n} \rightarrow 1$.

Suppose that a contraction mapping $f: C \rightarrow C$ is defined by

$$
f(x)=\frac{x+1}{2} \text { for all } x \in C
$$

and two sequences are defined by

$$
\alpha_{n, i}=\left\{\begin{array}{ll}
\frac{1}{n+1}, & i=0, \\
\left(1-\frac{1}{n+1}\right) \frac{1}{2^{i}}, & i \geq 1,
\end{array} \quad\left\{r_{n}\right\}=\left\{\frac{n+1}{2 n}\right\}, \quad n \geq 0, i \geq 1 .\right.
$$

It is easy to see that these sequences satisfy all of conditions in Theorem 1. Now, the following algorithm is presented for finding a proximal point $x \in F$ as in Theorem 1.

Algorithm 1 Proximal point algorithm via viscosity approximation method

Step 1. Choose $x_{0} \in C$ arbitrarily and let $n=0$.

Step 2. Compute the series value given by

$$
y_{n}=\sum_{i=1}^{\infty} \alpha_{n, i} S_{n} J_{r_{n}}^{A_{i}} x_{n} .
$$

Step 3. Update $x_{n+1}=\alpha_{n, 0} f\left(x_{n}\right)+y_{n}$.

Step 4. Put $n:=n+1$ and return to Step 1 .

In this experiment, we first test the effect of initial points on solution by choosing three initial points $x_{0}=50,10,-20$. The behavior of $x_{n}$ for Algorithm 1 is indicated in Figure 1.

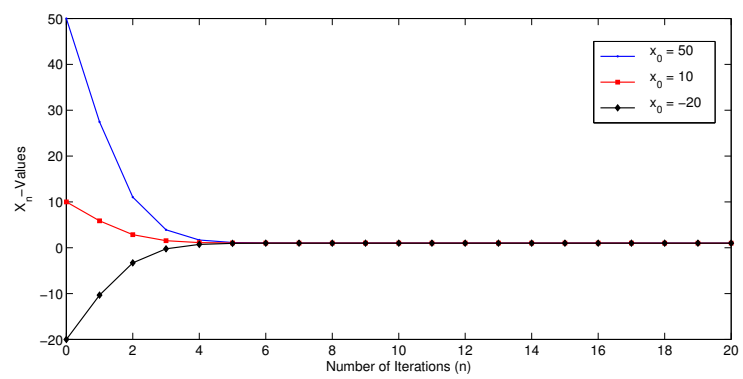

Figure 1. Behavior of $x_{n}$ for the different initial point $x_{0}=50,10,-20$.

This shows that for different initial points, each sequence $x_{n}$ converges to the same solution, i.e., $1 \in F$ as solution of the example. 
Next, we would examine the effect of $\alpha_{n, i}$ to rate of convergence and solution by setting three different sequences as follows;

$$
\begin{gathered}
\alpha_{n, i}=\left\{\begin{array}{ll}
\frac{1}{n+1}, & i=0 \\
\left(1-\frac{1}{n+1}\right) \frac{1}{2^{i}}, & i \geq 1
\end{array},\right. \\
\alpha_{n, i}^{\prime}=\left\{\begin{array}{ll}
\frac{1}{n+1}, & i=0 \\
\left(1-\frac{1}{n+1}\right) \frac{2}{3^{i}}, & i \geq 1
\end{array},\right. \\
\alpha_{n, i}^{\prime \prime}= \begin{cases}\frac{1}{3 n+1}, 1 \\
\left(1-\frac{1}{3 n+1}\right) \frac{1}{2^{i}}, & i \geq 1\end{cases}
\end{gathered}
$$

with the same initial point $x_{0}=30$ and $f(x)=(x+1) / 2$. By Algorithm $1, x_{n}$ converges to 1 and the behavior of $x_{n}$ is showed in Figure 2.

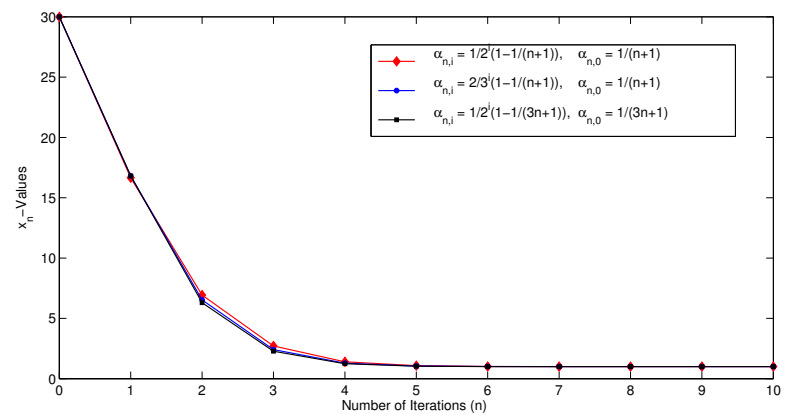

Figure 2. Behavior of $x_{n}$ for the different sequences $\alpha_{n, i}, \alpha_{n, i}^{\prime}$ and $\alpha_{n, i}^{\prime \prime}$.

Moreover, Figure 3 shows that by using Algorithm 1, the sequence $x_{n}$ also converges to 1 for the different contraction functions $f(x)=0.2(x+1), 0.5(x+1)$ and $0.8(x+1)$ with the same initial point $x=-10, \alpha_{n, 0}=\frac{1}{n+1}$ and $\alpha_{n, i}=$ $\left(1-\frac{1}{n+1}\right) \frac{1}{2^{i}}$. The presented results show that $f(x)=0.5(x+1)$ has the fastest rate of convergence.

The figures $1-3$ show that the sequence $x_{n}$ converge to the same value for the differences of initial points, sequences $\alpha_{n, i}$ and contraction functions. The values of the sequence $x_{n}$ on the test of the figures 1-3 are shown on Table 1. We note that the differences of initial points and parameter sequences $\alpha_{n, i}$ do not significant indicate the rates of convergence. However, the constants of contraction functions have likely effect on the rates of convergence. 


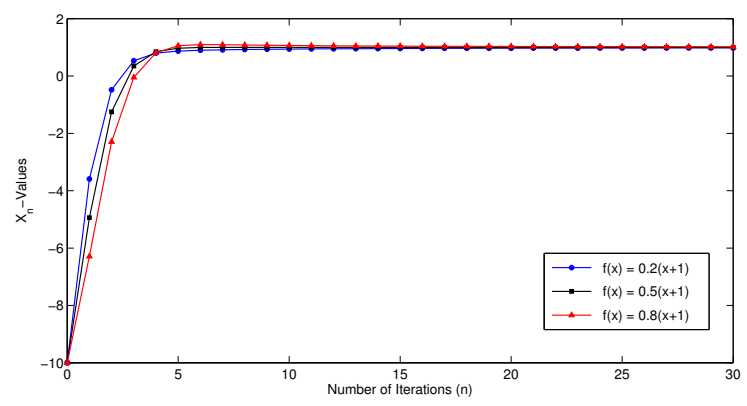

Figure 3. Behavior of $x_{n}$ for the different contraction functions $f(x)=0.2(x+1), 0.5(x+1)$ and $0.8(x+1)$.

Table 1. The values of the sequence $x_{n}$ on the test.

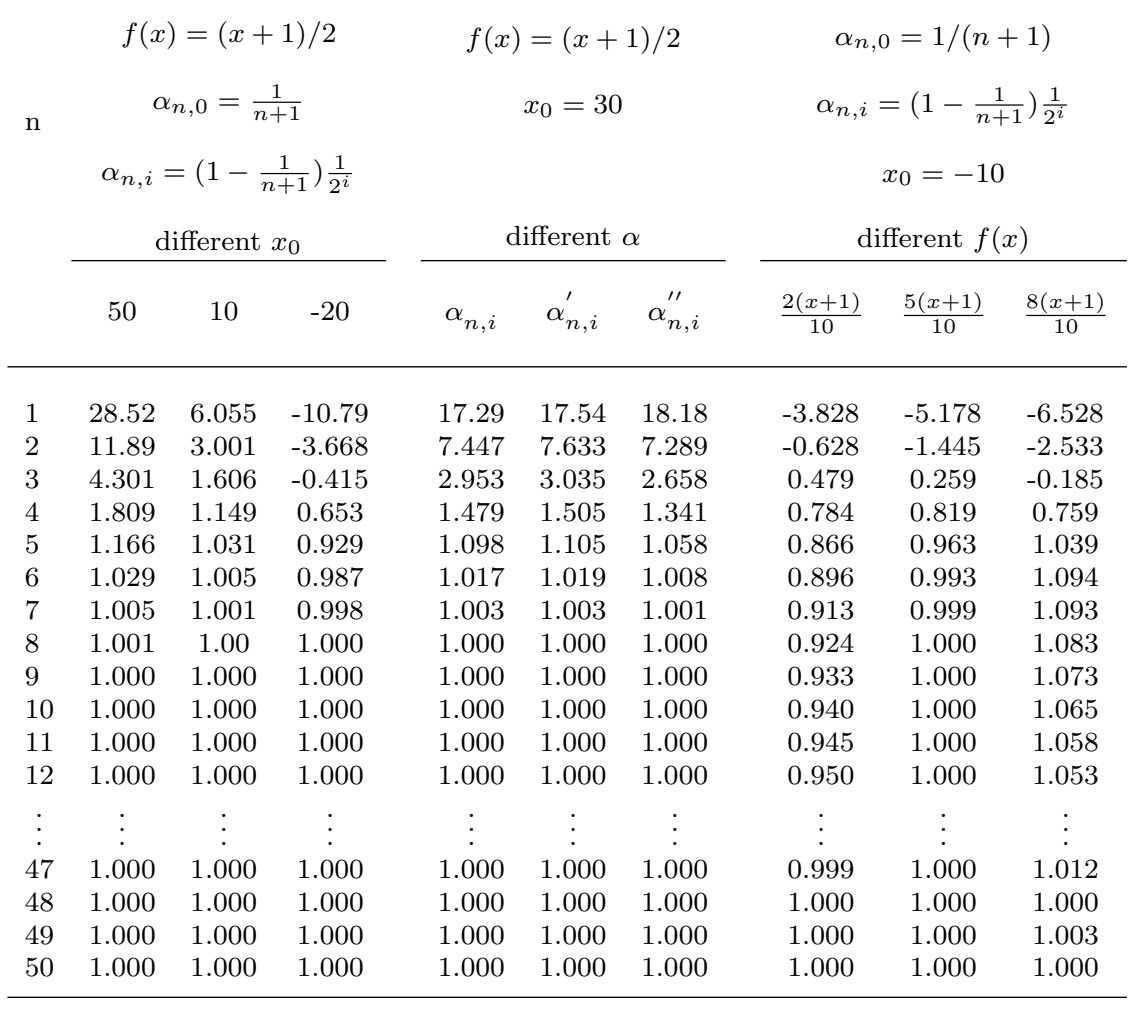

\section{Acknowledgments}

The first author would like to thank the Faculty of Education, Burapha University, for supporting by the Human Resource Development Scholarship. Moreover, this work the second author thank to the KMUTT Postdoctoral Fellow- 
ship 2014-2015 and the third author was supported by the Thailand Research Fund and the King Mongkut's University of Technology Thonburi

Grant No.RSA5780059.

\section{References}

[1] K. Aoyama, Y. Kimura, W. Takahashi and M. Toyoda. Approximation of common fixed points of a countable family of nonexpansive mappings in a Banach space. Nonlinear Analysis: Theory, Methods E Applications, 67(8):2350-2360, 2007. http://dx.doi.org/10.1016/j.na.2006.08.032.

[2] V. Barbu. Nonlinear Semigroups and Differential Equations in Banach Spaces. Editura Academiei Bucharest-Noordhoff, Leyden, 1976. http://dx.doi.org/10.1007/978-94-010-1537-0.

[3] F. E. Browder. Fixed-point theorems for noncompact mappings in Hilbert space. Proceedings of the National Academy of Sciences of the United States of America, 53(5):1100-1103, 1965.

[4] R.E. Bruck. Properties of fixed-point sets of nonexpansive mapping in Banach spaces. Transactions of the American Mathematical Society, 179:251-262, 1973. http://dx.doi.org/10.1090/S0002-9947-1973-0324491-8.

[5] C. Chidume. Geometric Properties of Banach Spaces and Nonlinear Iterations, volume 1965. Springer-Verlag London Limited, 2009. http://dx.doi.org/10.1007/978-1-84882-190-3.

[6] S.Y. Cho, X.L. Qin and L. Wang. Strong convergence of a splitting algorithm for treating monotone operators. Fixed Point Theory and Applications, 2014(1):94, 2014. http://dx.doi.org/10.1186/1687-1812-2014-94.

[7] I. Cioranescu. Geometry of Banach Spaces, Duality Mapping and Nonlinear Problems, volume 62. Springer Science \& Business Media, 1990. http://dx.doi.org/10.1007/978-94-009-2121-4.

[8] M. Eslamian. Rockafellars proximal point algorithm for a finite family of monotone operators. UPB scientific bulletin, Series A: applied mathematics and physics, 76(1):43-50, 2014.

[9] J.P. Gossez and E. Lami Dazo. Some geometric properties related to the fixed point theory for nonexpansive mappings. Pacific Journal of Mathematics, 40(3):565-573, 1972. http://dx.doi.org/10.2140/pjm.1972.40.565.

[10] J.S. Jung, Y.J. Cho and H. Zhou. Iterative processes with mixed errors for nonlinear equations with perturbed m-accretive operators in banach spaces. Applied mathematics and computation, 133(2):389-406, 2002. http://dx.doi.org/10.1016/S0096-3003(01)00239-9.

[11] S. Kitahara and W. Takahashi. Image recovery by convex combinations of sunny nonexpansive retractions. Topological Methods in Nonlinear Analysis, 2(2):333342, 1993.

[12] L. Liu. Ishikawa-type and mann-type iterative processes with errors for constructing solutions of nonlinear equations involving $\mathrm{m}$-accretive operators in Banach spaces. Nonlinear Analysis: Theory, Methods \& Applications, 34(2):307317, 1998. http://dx.doi.org/10.1016/S0362-546X(97)00579-8. 
[13] G. López, V. Martín-Márquez, F. Wang and H.K. Xu. Forward-backward splitting methods for accretive operators in Banach spaces. In Abstract and Applied Analysis, volume 2012. Hindawi Publishing Corporation, 2012. http://dx.doi.org/10.1155/2012/109236. Available from Internet: http://www . hindawi.com/journals/aaa/2012/109236/.

[14] H. Manaka and W. Takahashi. Weak convergence theorems for maximal monotone operators with nonspreading mappings in a hilbert space. Cubo A Math. J., 13(1):11-24, 2011.

[15] B. Martinet. Brève communication. régularisation d'inéquations variationnelles par approximations successives. ESAIM: Mathematical Modelling and Numerical Analysis - Modèlisation Mathèmatique et Analyse Numrique, 4(R3):154-158, 1970. Available from Internet: http://eudml.org/doc/193153.

[16] A. Moudafi. Viscosity approximation methods for fixed-points problems. Journal of Mathematical Analysis and Applications, 241(1):46-55, 2000. http://dx.doi.org/10.1006/jmaa.1999.6615.

[17] S. Reich. Weak convergence theorems for nonexpansive mappings in banach spaces. Journal of Mathematical Analysis and Applications, 67(2):274-276, 1979. http://dx.doi.org/10.1016/0022-247X(79)90024-6. Available from Internet: http://www.sciencedirect.com/science/article/pii/0022247X79900246.

[18] R.T. Rockafellar. Monotone operators and proximal point algorithm. SIAM journal on control and optimization, 14(5):877-898, 1976. http://dx.doi.org/10.1137/0314056.

[19] H.K. Xu. Inequalities in Banach spaces with applications. Nonlinear Analysis: Theory, Methods \& Applications, 16(12):1127-1138, 1991. http://dx.doi.org/10.1016/0362-546X(91)90200-K.

[20] H.K. Xu. Viscosity approximation methods for nonexpansive mappings. Journal of Mathematical Analysis and Applications, 298(1):279-291, 2004. http://dx.doi.org/10.1016/j.jmaa.2004.04.059. 